\title{
Weak Gravity Conjecture From Low Energy Observers' Perspective
}

\author{
Kazuyuki Furuuchi \\ Manipal Centre for Natural Sciences \\ Centre of Excellence, Manipal Academy of Higher Education \\ Dr. T.M.A. Pai Planetarium Building, Manipal 576104, Karnataka, India
}

\begin{abstract}
The Weak Gravity Conjecture (WGC) was proposed to constrain Effective Field Theories (EFTs) with Abelian gauge symmetry coupled to gravity. In this article, I study the WGC from low energy observers' perspective, and revisit the issue of to what extent the WGC actually constrains EFTs. For this purpose, for a given EFT, I introduce associated idealized low energy observers who only have access to the energy scale below the UV cut-off scale of the EFT. In the framework of EFT, there is a clear difference between the particles lighter than the UV cut-off scale and the particles which are heavier than the UV cut-off scale, as the lighter particles can be created below the UV cut-off scale while the heavier particles are not. This difference implies that the knowledge of the low energy observers on the stable heavy particles can be limited, as the availability of the stable heavy particles is determined by the environment prepared by some UV theory unknown to the low energy observers. The limitation of the knowledge of the low energy observers regarding the stable heavy particles whose mass is above the UV cut-off scale of the EFT leads to the limitation of the WGC for constraining EFTs. To illustrate these points in an example, I analyze a model proposed by Saraswat [1] which respects the WGC at high energy, but which may appear to violate the WGC for the low energy observers. Implications of the analysis to the bottom-up model buildings using EFTs are discussed.
\end{abstract}




\section{Contents}

1 Introduction 1

2 The WGC for low energy observers 5

2.1 Heavy Particle Effective Theory (HPET) . . . . . . . . . . . . 5

2.2 Low energy observers associated with an EFT and stable heavy particles . 7

2.3 Bound states in EFTs . . . . . . . . . . . . . . . . . . . . 8

2.4 The WGC for low energy observers . . . . . . . . . . . . . . . . . . . 9

2.5 When the magnetic WGC is satisfied, the electric WGC bound is automatically satisfied by charged particles which are lighter than the UV cut-off scale of the EFT . . . . . . . . . . . . . . . . . 111

2.6 Comments on monopoles . . . . . . . . . . . . . . . . . . . . 12

$2.7 \mathrm{BH}$ discharge arguments and mass of charged particles . . . . . . . . . . 13

3 The Saraswat model

3.1 The WGC under a spontaneous gauge symmetry breaking . . . . . . . . . 17

3.2 Implications to the bottom-up model buildings . . . . . . . . . . . . . . . . 29

4 Summary and discussions

A The weak electric WGC is not violated by spontaneous gauge symmetry breakings 36

\section{Introduction}

Since the vastness of the string landscape began to be revealed, criteria for efficiently separating out Effective Field Theories (EFTs) which cannot be derived from string theory [2] had been called for. One of the most extensively studied such criteria proposed so far is the Weak Gravity Conjecture (WGC) [3]. It requires that in an EFT with an unbroken Abelian ${ }^{1}$ gauge symmetry coupled to gravity, there exists a particle to which the electric Coulomb force acts stronger than the Newtonian gravitational force (electric WGC). Thus

\footnotetext{
${ }^{1}$ The extension of the WGC to non-Abelian gauge theories have been discussed [4, but in this work I focus on properties particular to Abelian gauge theories.
} 
the electric WGC in 4D requires the existence of a particle which satisfies $\mathrm{S}^{2}$

$$
\frac{g q M_{P}}{m} \gtrsim 1
$$

where $q$ is the charg $\complement^{3}$ of the particle and $m$ is its mass, $g$ is the gauge coupling constant and $M_{P}:=\sqrt{8 \pi G}$ is the reduced Planck mass ( $G$ is the Newton's constant). A physical motivation behind this conjecture is an expectation that extremal Black Holes (BHs) should be kinematically allowed to decay. By requiring the existence of a magnetic monopole to which the magnetic Coulomb force acts stronger than the Newtonian gravitational force (magnetic WGC), together with an estimate of the mass of the monopole, one obtains a bound on the UV cut-off scale $\Lambda$ of the EFT [3]:

$$
\Lambda \lesssim g M_{P}
$$

One of the motivations of the WGC was to use this bound to explain why extra-natural inflation had been difficult to realize in string theory [5]. As can be seen from its origin, aspects of the WGC as constraints on EFTs have been of great interests, though the WGC has also been extensively examined in string theory which is the leading candidate for a UV complete theory including quantum gravity. In this article, I revisit the issue of to what extent the WGC constrains EFTs.

The applicability of any EFT is limited by a certain UV cut-off scale $\Lambda$ above which the description by the EFT breaks down 4 Some new physical degrees of freedom must appear at $\Lambda$ to replace the description. In this framework of EFT, there is a clear distinction between particles lighter than $\Lambda$ and those which are heavier: The particles whose mass is below the UV cut-off scale of the EFT can be created within the EFT description, while creations of heavy particles whose mass is greater than $\Lambda$ cannot be described within the EFT. However, some stable heavy particles created at the energy above $\Lambda$ may remain at low energy. If these stable heavy particles are coupled to gauge fields, their interaction with the gauge particles could be of interest and they may be incorporated in EFT. Indeed, an EFT framework for describing such stable heavy particles heavier than the UV cut-off scale of the EFT had been developed in the past, one of the most important

\footnotetext{
${ }^{2}$ Throughout this article I will be concerned only with parametric relations, and I use $\sim$ to indicate that I are suppressing $\mathcal{O}(1)$ or even $\mathcal{O}(8 \pi)$ numerical factors.

${ }^{3}$ When I just write as charge, I mean the electric charge. For a magnetic charge, I will explicitly mention it.

${ }^{4}$ Without gravity, a Quantum Field Theory (QFT) can be UV complete, which corresponds to $\Lambda$ being infinity. With gravity, it is natural to assume that $\Lambda$ is finite and below the Planck scale. If one assumes asymptotically safe gravity, it may accommodate infinite $\Lambda$, but I will not consider such models.
} 
applications is in the study of heavy quark physics and known as Heavy Quark Effective Theory (HQET) $!^{5}$ I will give a brief review of the description of stable heavy particles in EFT in Sec. 2.1.

The point I would like to explore in this article is that it is possible that the low energy observers who only have access to the energy below the UV cut-off scale $\Lambda$ may or may not know the existence of these stable heavy particles ${ }^{6}$ The latter situation can happen if the stable heavy particles were not created with appreciable density, or diluted away after they were created. This is indeed a quite familiar setting in particle cosmology, and it could be of practical interests. For example, one of the motivations of cosmic inflation was to explain why in our universe we have not observed any magnetic monopole which was predicted by Grand Unified Theories (GUTs) [9, 10, 11]. After all, one of the ultimate goals of string theory is to explain the particle physics realized in our universe, and we have not observed any such relics from high energy so far in our universe (with possible exception of Dark Matters (DMs), however our knowledge on their nongravitational interactions is very limited, we only have experimental bounds). However, my interest here will be more on a matter of principle. Thus, for a given EFT, I will consider idealized low energy observers who can create and observe particles whose mass is below $\Lambda$. However, I do not always assume that the low energy observers know all the stable heavy particles whose mass is above the UV cut-off scale of the EFT, since how much they have been created is an input from the UV theory and is not under control of the low energy observers. This limitation of the knowledge of the low energy observers is a fundamental one which cannot be improved by the improvement of experimental techniques (even if one has a perfect detector, if there is no stable heavy particle left, it is impossible to detect it).7 Stable heavy particles might have been created in the early Universe or around a small high-temperature BH. However, these environments should be regarded as a kind of natural particle colliders, therefore if the observers are guaranteed to

\footnotetext{
${ }^{5}$ For a review of HQET, see for example [6, 7, 8,

${ }^{6}$ The possibility that charged particles which satisfy the bound 1.1 all have mass above the UV cut-off scale of EFT was briefly mentioned in the original article [3. However, the possibility that stable heavy particles whose mass is beyond the UV cut-off scale could be known to the low energy observers was not explored further, which I will do in the current article.

${ }^{7}$ If the density of the stable heavy particles left is small but finite, they can become detectable by the improvement of the sensitivity of the detector. However, such experimental practicalities are not the interest of the current article. In fact, what is relevant here is how much information of the stable heavy particles the low energy observers have. The environment prepared by the UV theory and experimental ability both limit this information, but the former is fundamental in a sense that it cannot be changed by the low energy observers.
} 
have access to stable particles heavier than the UV cut-off scale of the EFT produced from these environments, such observers should not be regarded as the low energy observers associated with the EFT. Thus I analyze the cases one by one, depending on which stable heavy particles are known to the low energy observers.

There are at least two reasons to take into account stable heavy particles when discussing the WGC. The first reason is that it is of practical interest to ask whether the WGC is satisfied in the low energy IR EFT when it arises from a UV EFT which satisfies the WGC. If the WGC can be violated in the IR EFT, this means that the constraining power of the WGC on low energy EFTs can be quite limited. However, if one assumes that the low energy observers associated with IR EFT do not know any particles whose mass is above the UV cut-off scale of IR EFT, then when all the charged particles have mass above the UV cut-off scale of the IR EFT, the WGC appears to be violated for the low energy observers associated with the IR EFT. For example, take Quantum ElectroDynamics (QED), and consider EFT obtained by integrating out degrees of freedom above the energy scale of the electron mass. There is no charged particle in the resulting EFT, and the electric WGC appears to be violated in this case, unless one considers the electrons whose mass is above the UV cut-off scale of the EFT. This example shows that in order to obtain meaningful constraints on EFTs from the WGC, one should take into account stable heavy particles whose mass is above the UV cut-off scale of the EFT under consideration. The second reason is that once one accepts the magnetic WGC, in particular the bound on the UV cut-off scale of EFTs (1.2), the electric WGC is automatically satisfied by any charged particles whose mass is below the UV cut-off scale, as will be explained in Sec. 2.5. This means that the electric WGC becomes a non-trivial question only when there is no charged particle whose mass is below the UV cut-off scale of the EFT under consideration.

The role of stable heavy particles in constraining EFTs by the WGC will be discussed in more detail in the main body. To illustrate the ideas, I take a simple model proposed by Saraswat [1] as an example. This is an interesting model which respects the WGC at high energy, but which can violate a version of the electric WGC at low energy. I further show that depending on the knowledge of the low energy observers on the stable heavy particles, there are cases in which the electric WGC appears to be violated. I also show that the magnetic WGC is respected in this model. To show this result, it is important to correctly identify the UV cut-off scale of the low energy EFT. For this purpose, I make detailed study of the internal structure of the monopole in this model which reveals the break-down of the low energy description. The break-down of the low energy description appears as fractional magnetic charges. The fractional magnetic charges would lead to 
observable Dirac strings which would be problematic. This is a break-down of the low energy description, and a new physics must enter to replace the low energy description. The new physics which makes the Dirac string unobservable here enters through the Aharonov-Bohm effect, which might have not been a common way for a new physics to appear in the models studied in the past.

After the detailed analysis of the role of the stable heavy particles in the Saraswat model, I draw general lessons for understanding actual constraints of the WGC on EFTs, which is the main theme of this article. Then, as an important example, I take the extranatural inflation model based on the 5D version of the Saraswat model and examine how the WGC constrains the model.

The organization of this article is as follows. In Sec. 2, I review some key aspects of the framework of EFT relevant for discussing the WGC. In particular, I first review how to describe stable heavy particles whose mass is above the UV cut-off scale of the EFT under consideration. This framework is referred to as Heavy Particle Effective Theory (HPET). Then, using HPET, I give a precise statement for what I mean by the low energy observers associated with an EFT in this article. The limitation of the knowledge of the low energy observers associated with EFTs regarding stable heavy particles and its implications to the WGC are analyzed. In Sec. 3, I take the model proposed by Saraswat to illustrate the points discussed in the previous section. Cases are classified depending on which stable heavy particles are known to the low energy observers, and are analyzed one by one. From this study, I draw general lessons for the bottom-up model buildings using EFTs. Then, the extra-natural inflation model based on the Saraswat model is studied in some detail. In Sec. 4, I summarize the results and discuss their implications towards the better understanding of the WGC.

\section{The WGC for low energy observers}

\subsection{Heavy Particle Effective Theory (HPET)}

I first review how to describe stable heavy particles whose mass is above the UV cutoff scale of an EFT, in the framework of EFT. The framework explained here had been applied in the study of heavy quark physics (Heavy Quark Effective Theory, HQET) (for a review of HQET, see for example [6, 7, 8]). Below, I explain the framework in the case where the stable heavy particle is a Dirac field, but the framework can also be used for particles with different spins [12, 13].

The starting idea for constructing the EFT for stable heavy particles is that in the 
infinite-mass limit, the velocity of the heavy particles are conserved. Therefore, the velocity is still a good quantum number for particles with heavy but finite mass. Thus one writes the four-momentum $p_{\mu}$ of a heavy particle state as [14, 15, 16, 17]

$$
p_{\mu}=m_{h} v_{\mu}+k_{\mu}
$$

where $m_{h}$ is the mass of the heavy particle and the four-velocity $v_{\mu}$ is a time-like unit vector which satisfies $v_{0}>0$ and $v \cdot v=1 . k_{\mu}$ is the so-called residual momentum and is a measure of how much the heavy particle is off-shell. The low energy observers are those who are interested in the low-energy processes which satisfy

$$
\left|k_{\mu}\right| \ll \Lambda<m_{h}
$$

where $\Lambda$ is the UV cut-off scale of the EFT.

The above idea is implemented at the action level as follows. Let $\Psi$ be a Dirac field with mass $m_{h}>\Lambda$, where $\Lambda$ is the UV cut-off scale of the EFT with an Abelian gauge symmetry. I start with the standard quadratic action for the Dirac field, which corresponds to a UV theory for the EFT to be obtained:

$$
S_{\Psi}=\int d^{4} x\left[\bar{\Psi} i \not D \Psi-m_{h} \bar{\Psi} \Psi\right]
$$

Here, the covariant derivative is given as

$$
D_{\mu} \Psi=\left(\partial_{\mu}+i g q A_{\mu}\right) \Psi
$$

where $g$ is the gauge coupling, $A_{\mu}$ is the Abelian gauge field and $q$ is the charge of the field $\Psi$ with respect to the Abelian gauge group.

To obtain the low energy EFT appropriate for describing processes with the residual momentum scale much lower than the UV cut-off scale (see (2.2)), one decomposes the field $\Psi$ into "light part" and "heavy part":

$$
\Psi=e^{-i m_{h} v \cdot x}\left(h_{v}+H_{v}\right)
$$

where

$$
h_{v}:=P_{+} \Psi, \quad H_{v}:=P_{-} \Psi, \quad P_{ \pm}:=\frac{1 \pm \psi}{2} .
$$

The field $h_{v}$ represents the light degrees of freedom whereas the field $H_{v}$ represents the heavy degrees of freedom, as can be seen shortly. Putting 2.5) into (2.2) gives

$$
S_{\Psi}=\int d^{4} x\left[\bar{h}_{v} i v \cdot D h_{v}-\bar{H}_{v}\left(i v \cdot D+2 m_{h}\right) H_{v}+\bar{h}_{v} i \not D_{\perp} H_{v}+\bar{H}_{v} i \not D_{\perp} h_{v}\right] .
$$


Here, $D_{\perp \mu}$ is the covariant derivative in the direction perpendicular to $v_{\mu}$ :

$$
D_{\perp \mu}:=\left(\delta_{\mu}^{\nu}-v_{\mu} v^{\nu}\right) D_{\nu} .
$$

From (2.7), one observes that $h_{v}$ is massless whereas $H_{v}$ has mass $2 m_{h}$ which is above the UV cut-off scale $\Lambda$. By integrating out the heavy field $H_{v}$, one obtains the effective action for the light degrees of freedom $h_{v}$. Using the classical equation of motion:

$$
(i v \cdot D+2 m) H_{v}=i \not D_{\perp} h_{v},
$$

one obtains the effective action for the light field $h_{v}$ :

$$
S\left[h_{v}\right]:=\int d^{4} x\left[\bar{h}_{v} i v \cdot D h_{v}+\bar{h}_{v} i \not D_{\perp} \frac{1}{2 m+i v \cdot D} i \not D_{\perp} h_{v}\right] .
$$

In the low energy regime 2.2 where the residual momentum is much smaller than the mass of the heavy particle, it would be appropriate to expand the second term in (2.10) in Taylor series:

$$
S\left[h_{v}\right]=\int d^{4} x\left[\bar{h}_{v} i v \cdot D h_{v}+\frac{1}{2 m_{h}} \sum_{n=0}^{\infty} \bar{h}_{v} i \not D_{\perp}\left(-\frac{i v \cdot D}{2 m_{h}}\right)^{n} i \not D_{\perp} h_{v}\right] .
$$

In the above, the low energy effective action for the light degrees of freedom $h_{v}$ was derived from the UV theory (2.3). On the other hand, in the current article, I will be interested in the case where the low energy observers do not know the UV theory. In this case, the coefficients of the higher order terms in the effective action (2.11) are not fixed theoretically, only experiments can fix them.

If one canonically quantize the action (2.7), $h_{v}$ corresponds to the field which annihilates a particle with four-velocity $v$, while $H_{v}$ corresponds to the field which annihilates an anti-particle with four-velocity $v$ (recall that I chose $v_{0}>0$ ). Thus after integrating out $H_{v}$, there is no degree of freedom which describes anti-particles. To obtain an EFT which describes stable heavy anti-particles, one replaces $v$ with $-v$ in the above procedures.

Below, I will refer to the EFT of stable heavy particles explained in this subsection as Heavy Particle Effective Theory, or HPET in brief.

\subsection{Low energy observers associated with an EFT and stable heavy particles}

To study the WGC from low energy observers' perspective, for a given EFT, I introduce a notion of low energy observers associated with the EFT. These low energy observers are 
assumed to have access to energy scale below the UV cut-off scale $\Lambda$ of the EFT. However, I do not always assume that these low energy observers know all the stable heavy particles whose mass is above $\Lambda$. This is because these heavy particles are not created below the energy scale $\Lambda$, thus their existence is controlled by a UV theory which describes physics above $\Lambda$, and it is not under the control of the low energy EFT.

The stable heavy particles whose mass is above the UV cut-off scale of the low energy EFT and which are known to the low energy observers should be included in the EFT in the framework of HPET, described in Sec. 2.1. Since I would like to consider idealized low energy observers who can get all the information contained in the EFT they are associated with, technically they can be identified with the associated EFT itself.

To summarize, what I mean by the low energy observers associated with an EFT in this article is:

EFT of fields whose mass is below the UV cut-off scale of the EFT, and HPET of stable heavy particles whose mass is above the UV cut-off scale of the EFT.

What I mean by "the stable heavy particles are known to the low energy observers" is:

The stable heavy particles are included in the EFT as HPET.

\subsection{Bound states in EFTs}

I briefly clarify my use of the terms "particle states" and "bound states" in EFTs. In EFTs, the internal structure of a bound state whose size is below the UV cut-off (length) scale $\sim 1 / \Lambda$ cannot be resolved, hence it is regarded as a point particle state in the EFT. Therefore, by bound state in EFT, I mean that the state has a spatial structure whose size is bigger than $1 / \Lambda$. In this article, I will use the term particle state exclusively for a point particle in EFTs, i.e. those state which do not have an internal structure larger than $1 / \Lambda$. Thus in my definition, bound states in EFT are not particle states. These definitions justify my earlier statements that in the framework of EFT, particles with mass larger than the UV cut-off scale of the EFT $\Lambda$ cannot be created. On the other hand, a bound state whose total energy is larger than $\Lambda$ can still be created, since the constituent particles can be created below $\Lambda$. 


\subsection{The WGC for low energy observers}

Consider an EFT in 4D with an unbroken Abelian gauge symmetry which couples to gravity. The electric WGC requires that there exists a particle whose charge $q$ and mass $m$ satisfies the bound

$$
\frac{g q M_{P}}{m} \gtrsim 1
$$

where $g$ is the coupling constant of the Abelian gauge theory and $M_{P}:=(8 \pi G)^{-\frac{1}{2}}$ is the reduced Planck mass ( $G$ is the Newton Constant). The normalization of the gauge coupling constant in Abelian gauge theories depends on the convention, even after one fixes the convention for the product $g q$. This point is not relevant for the electric WGC, as the gauge coupling constant $g$ and the charge $q$ appears in the combination $g q$. However, it will be crucial when stating the magnetic WGC, as I discuss shortly. This is in contrast with non-Abelian gauge theories in which the gauge field itself is charged and one can use it as a standard for the normalization of the gauge coupling constant. One consequence is that the notion of the gauge coupling constant of an Abelian gauge theory requires an existence of the minimal coupling to a charged particle. In other words, there is no physical meaning in the gauge coupling constant of an Abelian gauge theory if there is no charged particle in the theory. In the context of the WGC, the case in which there is no charged particle in the theory may be regarded as a special case in which the electric WGC is violated (this case is indeed a little bit special, in that the EFT should have some non-minimal coupling to the gauge field in order for the low energy observers to probe the existence of the gauge field). I will use the convention in which the gauge coupling constant of an Abelian gauge theory is normalized so that the smallest 8 charge is one. However, here enters the limitation of the low energy observers associated with the EFT I explained in Sec. 2.2 The idealized low energy observers know all the charge and the mass of the particles whose mass is below the UV cut-off scale $\Lambda$ of the EFT, but their knowledge of the charge and the mass of the stable heavy particles whose mass is above $\Lambda$ is limited and depends on the environment prepared by a UV theory. This means that the low energy observers may not know the existence of the particle whose charge is truly the smallest. Therefore, the more precise statement is that in this article I will normalize the gauge coupling constant of an Abelian gauge group in an EFT so that the smallest charge known to the low energy observers associated with the EFT is one. As discussed above, making clear the convention used for the Abelian gauge coupling is crucial when discussing the magnetic WGC, but it has been implicit in earlier literature and it seems

\footnotetext{
${ }^{8}$ Throughout this article, when I state a charge is small or big, what I am referring to is the absolute value of the charge.
} 
to have become a source of confusions in interpreting the Saraswat model, which I will discuss in detail in Sec. 3 .

There are several different versions of the electric WGC proposed in the past [3, 18, 19, 20, 21, 22, 23]. The difference is about which type of particle satisfies the bound 2.12. The weak version only requires that a particle with the largest charge-to-mass ratio satisfies the bound (2.12). The strong version requires that the lightest charged particle satisfies the bound. The sub-lattice version requires that some $k \in \mathbb{Z}$ and all $n \in \mathbb{Z}$, there exist particles of charge $q=k n$ with $g q M_{P} / m \gtrsim 1$. Since this version requires a tower of charged states whose masses are allowed to grow with charges, it seems more appropriate to examine it in the top-down approach starting from some fundamental theory like string theory. Since my interest is on the constraints on low energy EFTs from the WGC, I will not discuss this version further in this article. Another version, requiring that a particle with the smallest charge to satisfy the bound (2.12), is known to have counterexamples in string theory [3] and therefore is not of further interest.

The magnetic WGC requires that the magnetic Coulomb force acts stronger to a monopole with the smallest magnetic charge than the Newtonian gravitational force:

$$
\frac{g_{m} M_{P}}{m_{m 0}} \gtrsim 1
$$

Here, $m_{m 0}$ is the mass of the monopole with the smallest magnetic charge, and the magnetic gauge coupling constant $g_{m}$ is given in my convention

$$
g_{m}=\frac{2 \pi}{g}
$$

The Dirac quantization condition in this convention is

$$
q \cdot q_{m}=n, \quad(n: \text { integer })
$$

where $q$ is the electric charge and $q_{m}$ is the magnetic charge. $q=1$ is the smallest electric charge known to the low energy observers, and $q_{m}=1$ is the smallest magnetic charge still allowed for the given knowledge of the smallest electric charge (more discussions on this point will be given in Sec. 2.6). Notice that $q_{m}=1$ has been used in (2.13). The mass $m_{m 0}$ of the monopole with the smallest magnetic charge (still allowed) is estimated in the EFT as [3]:

$$
m_{m 0} \sim \frac{\Lambda}{g^{2}}
$$

Putting (2.14) and (2.16) into (2.13), a bound on the UV cut-off scale $\Lambda$ of the EFT is obtained [3]:

$$
\Lambda \lesssim g M_{P}
$$


The bound 2.17) can also be obtained by requiring that the monopole with the smallest magnetic charge is not a $\mathrm{BH}[3]$. The size of the monopole with the smallest magnetic charge is determined by the UV cut-off scale $\Lambda$ of an EFT. Assuming that it is bigger than the Schwarzschild radius:

$$
\frac{1}{\Lambda} \gtrsim \frac{m_{m 0}}{M_{P}^{2}},
$$

with the estimate of the mass $m_{m 0}$ of the monopole with the smallest magnetic charge (2.16), one again obtains the bound on the UV cut-off scale $\Lambda$ of the EFT (2.17).

It is important to notice that while the physical requirements which led to the bound on the UV cut-off did not depend on the convention, the expression (2.17) depends on the normalization convention of the gauge coupling constant. This was because the physical arguments which led to 2.17) involved the knowledge of the smallest magnetic charge which was dependent on the knowledge of the electric charges, which in turn was needed for a physically meaningful normalization of the gauge coupling constant. Making clear the convention used in (2.17) is crucial in models in which there exists a large hierarchy between charges of particles. The Saraswat model which I will study in Sec. 3 is an example of such models. As declared earlier, I use the convention in which the smallest charge known to the low energy observers associated with the EFT is normalized to one to state (2.17), which has an advantage that the Dirac quantization condition 2.15) becomes simple.

\subsection{When the magnetic WGC is satisfied, the electric WGC bound is automatically satisfied by charged particles which are lighter than the UV cut-off scale of the EFT}

After making clear the convention of the gauge coupling in the previous subsection, I can now state an important fact: When the bound on the UV cut-off scale of the EFT 2.17 holds, any charged particle whose mass is below the UV cut-off scale of the EFT automatically satisfies the electric WGC bound (2.12). To see this, let $q$ be the charge of a particle whose mass $m$ is below the UV cut-off scale $\Lambda$, i.e. $m<\Lambda$. Then it follows that

$$
\frac{g q M_{P}}{m}>\frac{g q M_{P}}{\Lambda} \gtrsim \frac{g q M_{P}}{g M_{P}}=q \geq 1 .
$$

The second inequality follows from the bound on the UV cut-off scale (2.17), while the last inequality follows from the convention used in this articl $e^{9}$ that the smallest charge known

\footnotetext{
${ }^{9}$ The physical result of course does not depend on the convention used. One just need to use the same convention which was used to state the magnetic WGC (2.17).
} 
to the low energy observers associated with the EFT under consideration is one. Thus any particle whose mass is below the UV cut-off scale $\Lambda$ satisfies the electric WGC bound (2.12), once $\Lambda$ satisfies the bound (2.17). To put it differently: When one does not consider charged particles whose mass is above the UV cut-off scale satisfies the bound (2.17), the electric WGC is trivially satisfied. On the other hand, when there is no charged particles below the UV cut-off scale of an EFT, unless one considers charged particles whose mass is above the UV cut-off scale, 2.17) itself cannot be stated, since there is no physically meaningful way to define the gauge coupling appearing in (2.17). These observations give strong motivation to study the case when some of the stable heavy charged particles are known to the low energy observers associated with EFTs, which is the main theme of the current article.

\subsection{Comments on monopoles}

From eq. (2.16) it follows that monopoles are much heavier than the UV cut-off scale $\Lambda$ in the perturbative regime $g \ll 1$. However, there is a difference between stable heavy electrically charged particles which satisfy the electric WGC bound (2.12) and monopoles. If no particles whose mass is below the UV cut-off scale $\Lambda$ of the EFT under consideration satisfies the electric WGC bound (2.12), the electric WGC predicts the existence of a charged particle whose mass is above $\Lambda$ and which satisfies the electric WGC bound. But other than the electric WGC itself, there is no reason to expect the existence of such particle. On the contrary, monopoles are classical solutions of Abelian gauge theories, therefore their existence is expected once the EFT with an Abelian gauge symmetry is given. Since the low energy observers are not guaranteed to know the true smallest charge, there is also a limitation in their knowledge of possible magnetic charges of the monopoles. Recall that I am using the convention that the smallest electric charge known to the low energy observers is normalized to one. In this convention, together with the normalization of the magnetic gauge coupling constant (2.14), the low energy observers predict the existence of magnetic monopoles with magnetic charge $q_{m}$ being non-zero integers:

$$
q_{m}= \pm 1, \pm 2, \pm 3, \cdots .
$$

However, if the true smallest electric charge is fractional, say $1 / 3$, then the magnetic charges allowed by the Dirac quantization condition are integers multiple of three:

$$
q_{m}= \pm 3, \pm 6, \pm 9, \cdots
$$


Therefore, what the low energy observers should expect is that the magnetic monopoles exist, and their magnetic charge should be some integer. But they should not expect that the magnetic monopoles with all integer charges must exist. In particular, they should not assume that the magnetic monopole with the unit charge must exist. This is because such prediction can fail if there exists a particle with a smaller charge than that known to the low energy observers, as in the example above. The limited knowledge of the low energy observers leads to a looser bound on the UV cut-off scale of the EFT under consideration, but a looser bound will not be wrong, it is just looser than the bound one can get from more precise knowledge.

If the low energy observers detect a monopole and get to know its magnetic charge, they can be at least sure for the existence of that magnetic charge. They can also constrain possible electric charges by the Dirac quantization condition. In the rest of this article, I focus on the cases in which monopoles are not observed by the low energy observers. The cases in which some monopoles have been detected by the low energy observers can be studied in a way similar to the analysis below.

\section{7 $\mathrm{BH}$ discharge arguments and mass of charged particles}

The discharge process of $\mathrm{BHs}$ provided an argument for the WGC [3]. I will examine it below. The main conclusion I draw here is that no restriction on the mass of the charged particles is put from this argument ${ }^{10}$ In particular, the mass of the charged particle can be above the UV cut-off scale of the EFT in which one examines the WGC. This conclusion provides another reason for including stable heavy particles whose mass is above the UV cut-off scale of the EFT into consideration when discussing the WGC.

The condition that charged BHs can release more charges than mass in Planck unit was one of the physical motivations behind the original proposal of the WGC [3]. In [3], the main concern was whether charged BHs were kinematically allowed to decay. The actual decay channels in semi-classical regime have been examined in [4] in the context of the WGC, extending the work of [24]. As the interest of the current article is the low energy observer's perspective, it would be adequate to assume that the UV cut-off scale of EFTs considered is at least few orders below the Planck scale, thus the semi-classical analysis will be sufficient.

Let me start with a brief review of the discharge process of BHs [24, 4]. To fix my

\footnotetext{
${ }^{10}$ The same conclusion was also stated in [1], but here I give more detailed analysis of the actual discharge processes.
} 
convention, I write down the Einstein action coupled to an Abelian gauge field:

$$
S=\frac{1}{16 \pi G} \int d^{4} x \sqrt{-\operatorname{det}\left[g_{\mu \nu}\right]}\left[R-\frac{1}{4} F_{\mu \nu} F^{\mu \nu}\right] .
$$

Here, the cosmological constant is assumed to be negligible. Then, assuming naturalness, ${ }^{11}$ the action 2.22 gives the leading terms in the low energy approximation. Charged BHs are described by the Reissner-Nordström (RN) BH solution:

$$
\begin{gathered}
d s^{2}=-f(r) d t^{2}+\frac{1}{f(r)} d r^{2}+r^{2} d \Omega_{2}^{2}, \quad A_{0}(r)=\frac{g Q}{r}, \\
f(r)=1-\frac{2 G M}{r}+\frac{G g^{2} Q^{2}}{r^{2}}
\end{gathered}
$$

where $Q$ and $M$ are the charge and the mass of the $\mathrm{BH}$, respectively. I require that the charge and the mass of BHs satisfy the BPS bound 12

$$
\frac{g Q M_{P}}{M} \lesssim 1
$$

The radius of the outer horizon of the black hole is given by

$$
r_{+}=G M+\sqrt{(G M)^{2}-G g^{2} Q^{2}} .
$$

Notice that $G M \leq r_{+} \leq 2 G M$, so $r_{+}$is of the order of $G M$.

Let $q$ and $m$ be the charge and the mass of a particle which satisfy the electric WGC bound (2.12). When the temperature of the $\mathrm{BH}$ is high compared with the mass of the charged particle, Hawking radiation is the dominant process for the discharge of the $\mathrm{BH}$, while when the temperature is lower than the mass, the Schwinger pair-production is the dominant process.

\section{Discharge by Hawking radiation}

I first consider the case where the Hawking temperature $T_{H}$ of a $\mathrm{BH}$ is higher than the mass $m$ of the a particle with charge $q$ satisfying the electric WGC bound (2.12):

$$
T_{H} \gtrsim m
$$

Since for a $\mathrm{BH}$ with given mass $M$

$$
\frac{1}{G M} \gtrsim T_{H},
$$

\footnotetext{
${ }^{11}$ For a review of naturalness in EFTs, see for example [25].

${ }^{12}$ This requirement is motivated by the cosmic censorship hypothesis, which may be related to the WGC [26].
} 
I obtain

$$
\frac{1}{G M} \gtrsim m
$$

which can be rewritten as

$$
M \lesssim \frac{M_{P}^{2}}{m}
$$

The above was a condition for the $\mathrm{BH}$ to efficiently emit particles with mass $m$. In order for the charge to be emitted efficiently, the chemical potential should be greater than the mass of the charged particle. This condition gives

$$
\frac{g^{2} q Q}{r_{+}} \gtrsim m
$$

Since $r_{+} \sim G M \sim M / M_{P}^{2}$ and $g Q M_{P} \leq M$ from (2.24), it follows that

$$
\frac{g q M_{P}}{m} \gtrsim 1
$$

This is nothing but the electric WGC bound (2.12), which I already assumed so that the charged particle can take more charge than mass in Planck unit from the BH.

\section{Discharge by Schwinger pair-productions}

Next I consider the case when the Hawking temperature of the $\mathrm{BH}$ is below the mass of a particle that satisfies the electric WGC bound (2.12):

$$
T_{H} \lesssim m
$$

where $m$ is the mass of the particle. The dominant process in this case is the Schwinger pair-production.

A RN BH discharges appreciably through the pair creations of charged particles with charge $q$ and mass $m$ by the Schwinger pair-productions when [24, 4]

$$
m^{2} \lesssim \frac{g^{2} q Q}{r_{+}^{2}} .
$$

Since $r_{+} \sim G M \sim M / M_{P}^{2}$ and $g Q M_{P} \lesssim M$ by (2.24), it follows that

$$
m^{2} \lesssim g q \frac{M_{P}^{3}}{M}
$$

This can be rewritten as

$$
M \lesssim \frac{M_{P}^{2}}{m}\left(\frac{g q M_{P}}{m}\right)
$$




\section{The bound on the mass of BHs which can efficiently discharge}

From 2.29) and 2.35), it follows that in order for a $\mathrm{BH}$ to discharge efficiently, there are bounds on the mass of the $\mathrm{BH}$ [24]. If one requires $\mathrm{RN}$ BHs with arbitrary large mass should decay efficiently, one need to have a charged particle with arbitrary large ratio $M_{P} / m$. This directly follows from $(2.29)$ when the Hawking radiation is the dominant process. For the case when the Schwinger pair-production is the dominant process for discharge, note that $g q$ cannot be infinite, i.e. the notion of an infinite charge would be ill-defined, and in fact $g q \lesssim 1$ is required for the gauge theory to be in the perturbative regime, which I will assume in the following. Then, from (2.35) the condition that BHs with arbitrary mass can decay via the Schwinger process requires arbitrary large ratio $M_{P} / m$. This is equivalent to requiring the existence of a massless charged particle, which appears to be a too strong constraint which even QED in our universe does not satisfy. This would not be what the WGC requires on EFTs. Notice that the BHs with arbitrary mass should discharge efficiently led to a requirement for IR of the theory (i.e. the smallest mass of the charged particles which satisfy the electric WGC bound is relevant) rather than UV.

If the WGC does not require BHs with arbitrary mass to decay efficiently, there would be two alternative possibilities for the requirement for the $\mathrm{BH}$ discharge processes in the argument for the WGC:

1. BHs need not decay efficiently. Both Hawking radiation and Schwinger pair-production can be interpreted as quantum tunneling [27, 28], and as such as long as the final state is kinematically allowed, the $\mathrm{BH}$ has a probability to decay, though it is exponentially suppressed and the time scale required would easily exceed the age of the Universe.

2. Only BHs smaller than a certain scale, possibly a certain particle physics scale, are required to decay efficiently.

The first possibility does not impose any further condition on the mass of the charged particle which satisfy the electric WGC bound (2.12). I think this is indeed reasonable.

The second possibility requires an extra assumption about what should be the "certain scale" which bounds the size of the BHs which should decay efficiently. While some arguments to avoid remnant: ${ }^{13}$ may provide such an additional input, I have not come up with a reasonable candidate for such a scale. To get the feeling of the scales involved, consider QED with the electron as the lightest charged particle, which obviously satisfies

\footnotetext{
${ }^{13}$ For a review of BH remnants and the pathologies they may introduce, see for example [29].
} 
the electric WGC bound (2.12). Then, the bound 2.35) gives $M \lesssim 10^{5} M_{\odot}$, where $M_{\odot}$ is the solar mass [24]. However, this astrophysical mass is due to the large hierarchy between the Planck scale and the mass of the electron. In a model in which the charged particles relevant for the discharge of a $\mathrm{BH}$ have mass just few orders below the Planck scale, the mass and the size of BHs which can efficiently discharge can easily fall in the realm of particle physics.

In the rest of this article, I take the first possibility, i.e. I assume that BHs only need to be kinematically allowed to discharge, the actual process for the discharge need not be efficient. Then, as stated above, there is no constraint on the mass of the charged particles which satisfy the electric WGC bound 2.12). An important consequence is that the charged particles relevant for BH discharge need not have mass below the UV cut-off scale of an EFT under consideration. The above observation supports the idea that one should take into account stable heavy particles whose mass is above the UV cut-off scale of EFTs when studying constraints of the WGC to low energy EFTs.

\section{The Saraswat model}

\subsection{The WGC under a spontaneous gauge symmetry breaking}

In an interesting article [1], Saraswat proposed a simple model in which the WGC is respected at high energy, but after a spontaneous gauge symmetry breaking the strong version of the electric WGC can be violated at low energy $[4$ The embedding of the Saraswat model to string theory was examined in [31]. Below I will further examine the cases where the weak version of the electric WGC is not actually violated, but appears to be violated for the low energy observers associated with the low energy EFT, due to the limitation of their knowledge regarding the stable heavy particles. I will also show that the magnetic WGC is not violated at low energy in this model.

The Saraswat model has two Abelian gauge groups which I denote $U(1)_{A}$ and $U(1)_{B}$. Following [1, for simplicity I take $g_{A}=g_{B}=g$, where $g_{A}$ and $g_{B}$ are the coupling constants of the gauge group $U(1)_{A}$ and $U(1)_{B}$, respectively. It is straightforward to generalize the arguments below to the case $g_{A} \neq g_{B}$. As explained in the previous section, these gauge couplings are normalized so that the smallest charge known to the low energy observers associated with the EFT is one. With this convention, I introduce fields $\psi_{A}$ and $\psi_{B}$ which have the charge vectors $\vec{q}_{A}=(1,0)$ and $\vec{q}_{B}=(0,1)$, respectively. Here, the first entry refers to the charge with respect to the $U(1)_{A}$ gauge group, and the second entry

\footnotetext{
${ }^{14}$ The low energy theory is similar to the one studied in 30 .
} 
refers to the charge with respect to the $U(1)_{B}$ gauge group. In addition, I introduce a Higgs field $H$ which has a charge vector $\vec{q}_{H}=(Z, 1)$, where $Z$ is an integer. I assume that all the particles appearing in this EFT have mass below the UV cut-off scale $\Lambda$ of the EFT:

$$
m_{A}, m_{B}, m_{H}<\Lambda
$$

where $m_{A}$ and $m_{B}$ are the masses of the $\psi_{A}$ and $\psi_{B}$, respectively, and $m_{H}$ is the mass of the Higgs particle in the vacuum (to be explained below). I further assume that $\psi_{A}$ and $\psi_{B}$ are the lightest particles among those charged with respect to $U(1)_{A}$ and $U(1)_{B}$, respectively. Since the Higgs field is charged under both $U(1)_{A}$ and $U(1)_{B}$, this assumption implies

$$
m_{A}, m_{B}<m_{H}
$$

I require that the model satisfies the strong electric- and the magnetic WGC to begin with, and examine what happens below the spontaneous gauge symmetry breaking scale. When there are multiple $U(1)$ gauge groups, the bounds of the WGC need to be generalized accordingly [18]. To describe the WGC bounds, I define the charge-to-mass ratio vectors as follows:

$$
\vec{z}_{A}=\frac{g \vec{q}_{A} M_{P}}{m_{A}}, \quad \vec{z}_{B}=\frac{g \vec{q}_{B} M_{P}}{m_{B}} .
$$

The strong electric WGC means the the electric WGC is satisfied by the lightest charged particles $\psi_{A}$ and $\psi_{B}$. Then, the electric WGC requires that a unit disk, which represents possible charge-to-mass ratio of BHs constrained by the BPS bound (2.24), is contained in the convex hull spanned by the vectors $\pm \vec{z}_{A}$ and $\pm \vec{z}_{B}$ [18] (see also Appendix A). This requirement gives the conditions 15

$$
m_{A}, m_{B} \lesssim \frac{g M_{P}}{\sqrt{2}}
$$

The bound on the UV cut-off scale of the EFT becomes

$$
\Lambda \lesssim \frac{g M_{P}}{\sqrt{2}}
$$

where $\Lambda$ is the UV cut-off scale above which the description by this EFT breaks down. Note that the assumptions made above are not all independent: From the assumption (3.1) and the bound on the UV cut-off (3.5), the electric WGC 3.4 automatically follows, as explained in Sec. 2.5.

\footnotetext{
${ }^{15}$ In this article I have been neglecting $\mathcal{O}(1)$ numerical factors, therefore $1 / \sqrt{2}$ in $(3.4)$ and (3.5) should be understood only relative to 2.12 .
} 
After the Higgs field acquires a vacuum expectation value, only a linear combination of the gauge fields remains massless. I call the gauge field of the gauge group $U(1)_{A}$ as $A_{\mu}$, and the gauge field of the gauge group $U(1)_{B}$ as $B_{\mu}$. Then, a convenient basis for identifying the massless combination of the gauge fields is obtained by the linear transformation:

$$
\left(\begin{array}{c}
\tilde{A}_{\mu} \\
\tilde{B}_{\mu}
\end{array}\right)=\left(\begin{array}{cc}
\cos \gamma & \sin \gamma \\
-\sin \gamma & \cos \gamma
\end{array}\right)\left(\begin{array}{c}
A_{\mu} \\
B_{\mu}
\end{array}\right)
$$

where

$$
\cos \gamma:=\frac{Z}{\sqrt{1+Z^{2}}}, \quad \sin \gamma:=\frac{1}{\sqrt{1+Z^{2}}} .
$$

Let $v$ be the vacuum expectation value of the Higgs field. Then, $\tilde{A}_{\mu}$ acquires a mass $m_{V}=g \sqrt{1+Z^{2}} v$ while $\tilde{B}_{\mu}$ remains massless. I will refer to the unbroken $U(1)$ gauge

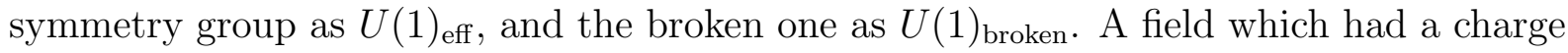
vector $(m, n)$ in the original basis has $(m \cos \gamma+n \sin \gamma,-m \sin \gamma+n \cos \gamma)_{\text {new }}$ in the new basis after the linear transformation (3.6). For example, the field $\psi_{A}$ which had $(1,0)$ in the original basis has $(\cos \gamma,-\sin \gamma)_{\text {new }}$ in the new basis (before rescaling the gauge coupling constant following my convention, which will be done below). When $Z \gg 1, \sin \gamma \ll 1$, i.e. $\psi_{A}$ has a very small charge (before rescaling the gauge coupling constant) with respect to $U(1)_{\text {eff }}$. Such small charge, which will be translated into small gauge coupling constant after the rescaling following the convention for the gauge coupling constant, is a potential source for a violation of the electric WGC. I will study the consequence of this small charge in some detail below.

Now, I examine the WGC from the viewpoint of the low energy observers associated with the EFT with unbroken $U(1)_{\text {eff }}$ gauge symmetry. I first need to identify what is the appropriate EFT to state the WGC with respect to the $U(1)_{\text {eff }}$ gauge symmetry. For the

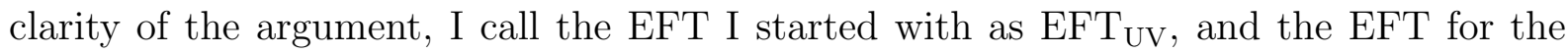

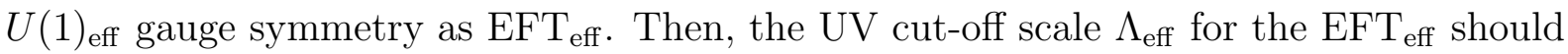
be at least below the gauge symmetry breaking scale which is characterized by the mass of the massive gauge field, since otherwise $\mathrm{EFT}_{\mathrm{UV}}$ with the $U(1)_{A} \times U(1)_{B}$ gauge symmetry becomes the appropriate description. Hence

$$
\Lambda_{\mathrm{eff}}<m_{V}=g \sqrt{1+Z^{2}} v .
$$

However, I will obtain a tighter bound on $\Lambda_{\text {eff }}$ from the analysis of the size of the monopole of $U(1)_{\mathrm{eff}}$ in this model below.

It is important to notice that the WGC for $\mathrm{EFT}_{\mathrm{UV}}$ and that for $\mathrm{EFT}_{\text {eff }}$ are different, for example, the gauge groups and the gauge coupling constants are different. 
As I explained in Sec. 2.5, when the mass of the field $\psi_{A}\left(\right.$ or $\left.\psi_{B}\right)$ is below the UV cut-off scale of EFT eff $_{\text {i.e. }} m_{A}<\Lambda_{\text {eff }}$ (or $m_{B}<\Lambda_{\text {eff }}$ ), the electric WGC bound is automatically

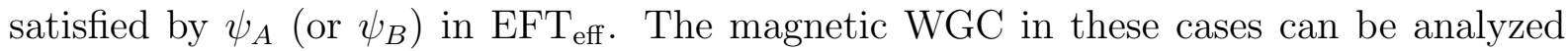
in the same way as in the case 1 below. In the following I examine the remaining case $m_{A}, m_{B}>\Lambda_{\text {eff }}$. The Higgs particles can be stable when $m_{H}<Z m_{A}+m_{B}$, which could mostly be the case when $Z \gg 1$. For simplicity, I restrict myself to the cases where the low energy observers associated with $\mathrm{EFT}_{\text {eff }}$ do now know the existence of the Higgs particles. It is straightforward to include the case where the existence of the Higgs particles is

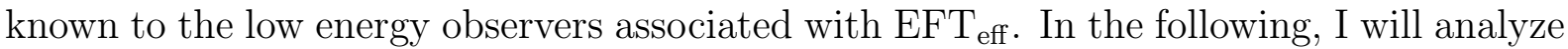
the cases differ in which particles are known to the low energy observers associated with $\mathrm{EFT}_{\text {eff }}$.

\section{Case 1: Both $\psi_{A}$ and $\psi_{B}$ are known to the low energy observers associated with EFT $_{\text {eff }}$}

In this case, the $\psi_{A}$ particles have the smallest charge with respect to $U(1)_{\text {eff }}$ among the charged particles which are known to the low energy observers associated with the EFT eff $_{\text {. }}$ Thus in my convention that the smallest charge known to the low energy observer is one, the gauge coupling constant $g_{\text {eff }}$ for the $U(1)$ eff gauge group is given as

$$
g_{\mathrm{eff}}=g \sin \gamma .
$$

As mentioned earlier, it is crucial to identify the UV cut-off scale $\Lambda_{\text {eff }}$ of EFT $_{\text {eff }}$ correctly in order to discuss the magnetic WGC. As it turns out, for this purpose it is important to first understand how the monopole of $U(1)$ eff is described in the UV theory, i.e. EFT $_{\text {UV }}$. As found in [1], a monopole with unit magnetic charge with respect to $U(1)_{\text {eff }}$ is constructed in EFT $_{\mathrm{UV}}$ from $Z$ monopoles of $U(1)_{B}$ and one anti-monopole of $U(1)_{A}$, connected by Nielsen-Olesen flux tubes [32, 33] (see Fig. 1). Indeed, with the magnetic gauge coupling (2.14), the magnetic charge vector for this system in the new basis can be read off using the linear transformation $(3.6)$ as

$$
g_{m}\left(\begin{array}{cc}
\cos \gamma & \sin \gamma \\
-\sin \gamma & \cos \gamma
\end{array}\right)\left(\begin{array}{c}
-1 \\
Z
\end{array}\right)=\frac{2 \pi}{g} \sqrt{1+Z^{2}}\left(\begin{array}{l}
0 \\
1
\end{array}\right)_{\text {new }}=\frac{2 \pi}{g_{\mathrm{eff}}}\left(\begin{array}{l}
0 \\
1
\end{array}\right)_{\text {new }}
$$

The radius of the flux tube is $\sim 1 / m_{V}$, and the tension is $\sim v^{2}$. Here, as in [1], I assumed that the mass of the Higgs field is greater than the mass $m_{V}=g \sqrt{1+Z^{2}} v$ of the massive gauge field, in which case the symmetry-breaking vacuum acts like a type II superconductor [32, 33]. This assumption is consistent with the assumptions I made 


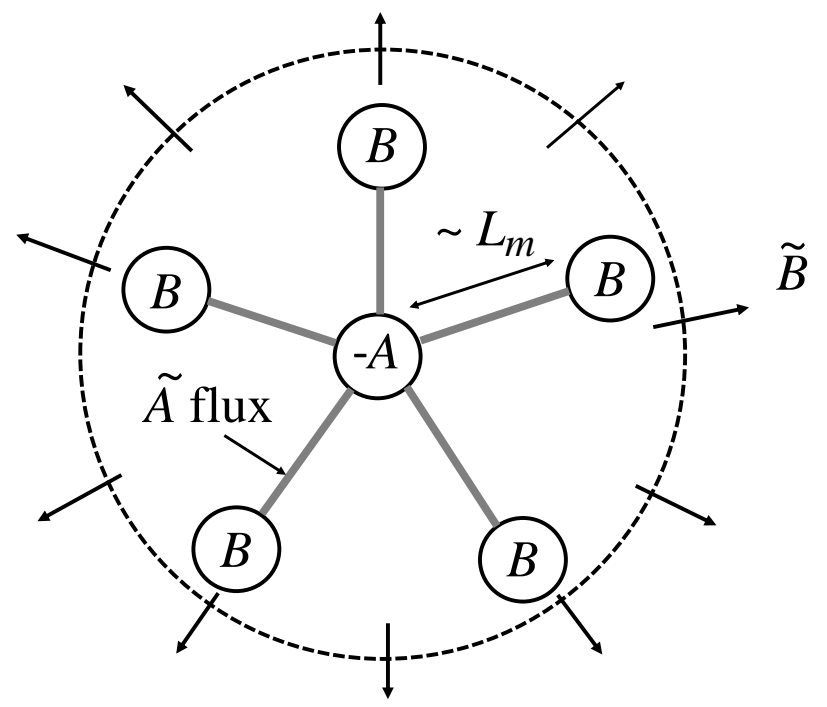

Figure 1: A schematic picture of a monopole of $U(1)_{\text {eff }}$ with unit magnetic charge. It consists of one anti-monopole of $U(1)_{A}$ and $Z$ monopoles of $U(1)_{B}$, connected by the Nielsen-Olesen flux tubes with $1 /\left(1+Z^{2}\right)$ of the $U(1)_{\text {broken }}$ flux which I indicate by $\tilde{A}$ inside of each [1]. Here, the flux number is normalized so that that from a monopole with unit magnetic charge is normalized to one.

and will make later, $v, m_{H}<\Lambda$ and $g Z \lesssim 1$. The size $L_{m}$ of the system is estimated from a balance between the energy in the flux tubes $\sim Z v^{2} L_{m}$ and the magnetic repulsive potential energy between the monopoles $\sim Z^{2}\left(\frac{2 \pi}{g}\right)^{2} \frac{1}{L_{m}}$ :

$$
L_{m} \sim \frac{\sqrt{Z}}{g v} .
$$

Here, I did not take into account the gravitational force, as it will turn out to be subdominant due to the magnetic WGC being satisfied. The mass $m_{m 0}$ of the monopole with unit magnetic charge with respect to the gauge group $U(1)_{\text {eff }}$ is estimated from the energy in the flux-tubes and the magnetic potential:

$$
m_{m 0} \sim \frac{Z^{\frac{3}{2}} v}{g} .
$$

Since the internal structure of the monopole of $U(1)_{\text {eff }}$ cannot be described by $\mathrm{EFT}_{\text {eff }}$, the size of the monopole $L_{m}$ represents the scale where the description by $\mathrm{EFT}_{\text {eff }}$ breaks down. In fact, consider the low energy observers who have access to energy scale beyond $1 / L_{m}$ but below $m_{V}$. Since they can probe beyond the length scale shorter than $L_{m}$, they can observe the monopoles of $U(1)_{B}$ and anti-monopole of $U(1)_{A}$ individually using 


\begin{tabular}{|l|l|l|}
\hline particle & charge in $U(1)_{A} \times U(1)_{B}$ & charge in $U(1)_{\text {broken }} \times U(1)_{\text {eff }}$ \\
\hline$\psi_{A}$ & $($ e) $g(1,0)$ & (e) $g_{\text {eff }}(Z,-1)_{\text {new }}$ \\
\hline$\psi_{B}$ & $($ e) $g(0,1)$ & (e) $g_{\text {eff }}(1, Z)_{\text {new }}$ \\
\hline Higgs $H$ & $($ e) $g(Z, 1)$ & (e) $g_{\text {eff }}\left(1+Z^{2}, 0\right)_{\text {new }}$ \\
\hline$U(1)_{A}$ anti-monopole & $\left(\right.$ m) $\frac{2 \pi}{g}(-1,0)$ & (m) $\frac{2 \pi}{g_{\text {eff }}}\left(-\frac{Z}{1+Z^{2}}, \frac{1}{1+Z^{2}}\right)_{\text {new }}$ \\
\hline$U(1)_{B}$ monopole & (m) $\frac{2 \pi}{g}(0,1)$ & (m) $\frac{2 \pi}{g_{\text {eff }}}\left(\frac{1}{1+Z^{2}}, \frac{Z}{1+Z^{2}}\right)_{\text {new }}$ \\
\hline
\end{tabular}

Table 1: (e) and (m) indicate the electric- and magnetic charge, respectively. I also associated the gauge couplings to the charges. The subscript "new" indicates that they are in the new basis of the charge vector (the charge vector in $U(1)_{\text {broken }} \times U(1)_{\text {eff }}$ basis).

the gauge field of $U(1)_{\text {eff }}$. Each monopole of $U(1)_{B}$ has magnetic charge $Z /\left(1+Z^{2}\right)$ with respect to $U(1)_{\text {eff }}$, while the anti-monopole of $U(1)_{A}$ has $1 /\left(1+Z^{2}\right)$ (see Table 1 , which can be calculated as in (3.10) $)$. They can also observe the attractive force between the monopoles of $U(1)_{A}$ and $U(1)_{B}$ due to the tension of the flux tubes, although they cannot resolve the radial size of the flux tube $\sim 1 / m_{V}$. The monopoles with fractional magnetic charges with respect to $U(1)_{\text {eff }}$ must have observable Dirac strings unless some new physics need to appear and prevent the Dirac strings from being observed: Stable heavy particles whose mass is above the UV cut-off of the EFT can be described in the framework of EFT as reviewed in 2.1, and once $\Psi_{A}$ and $\Psi_{B}$ are described in this way, Dirac string becomes observable through the Aharonov-Bohm Effect, unless new physics is introduced above $L_{m}$. Indeed, in the current case, I started from the UV theory $\mathrm{EFT}_{\mathrm{UV}}$, and in $\mathrm{EFT}_{\mathrm{UV}}$, which is a new physics for $\mathrm{EFT}_{\text {eff }}$, the fractional magnetic charges of the monopoles are consistent with the Dirac quantization, as I explain below. To make Dirac quantization with respect to $U(1)_{\text {eff }}$ consistent, the Dirac strings of $U(1)_{\text {eff }}$ and $U(1)_{\text {broken }}$ should be combined appropriately ${ }^{16}$ Actually, the appropriate combination is the Dirac strings of the original gauge group, $U(1)_{A} \times U(1)_{B}$, see Fig. 2, 4 . The Dirac string of $U(1)_{\text {eff }}$ for the monopole of $U(1)_{B}$ corresponds to that for the fractional magnetic charge $Z /\left(1+Z^{2}\right)$ (Table 1). This should be combined with the Dirac string of $U(1)_{\text {broken }}$ which corresponds to the fractional magnetic charge $1 /\left(1+Z^{2}\right)$. When a $\psi_{A}$ particle whose charge in $U(1)_{\text {broken }} \times U(1)_{\text {eff }}$ basis is $(Z,-1)_{\text {new }}$ travels around the combined Dirac string, the Aharonov-Bohm phase from the coupling to $U(1)_{\text {eff }}$ gauge field cancels with

\footnotetext{
${ }^{16} \mathrm{An}$ analogous argument has been given in order to make Dirac quantization condition consistent with the fractional electric charge of quarks with a Dirac monopole with unit magnetic charge. For further readings, see for example Sec. 5.1 of Ref. 34].
} 




$$
g_{\mathrm{eff}} Z \cdot \frac{2 \pi}{g_{\mathrm{eff}}} \frac{1}{1+Z^{2}}+g_{\mathrm{eff}}(-1) \cdot \frac{2 \pi}{g_{\mathrm{eff}}} \frac{Z}{1+Z^{2}}=0 .
$$

Hence, the $\psi_{A}$ particle cannot probe the combined Dirac string. Similarly, the Dirac string from the anti-monopole of $U(1)_{A}$ should be a combination of that from $-Z /\left(1+Z^{2}\right)$ of magnetic charge of $U(1)_{\text {broken }}$ and $1 /\left(1+Z^{2}\right)$ of magnetic charge of $U(1)_{\text {eff }}$. One can check that neither $\psi_{A}$ nor $\psi_{B}$ can probe the combined Dirac string from the monopole of $U(1)_{A}$ or that from the monopole of $U(1)_{B}$. One can choose a different gauge for the unbroken gauge group $U(1)_{\text {eff }}$. However, only the Dirac string which corresponds to that for the unit magnetic charge monopole can be deformed by the gauge transformation (Fig. 5). Such gauge transformation only changes the Aharonov-Bohm phase of the $\psi_{A}$ particle traveling around the Dirac string by $2 \pi$, which is not observable. Of course, when I considered the monopoles of $U(1)_{A}$ and $U(1)_{B}$, I already took into account the Dirac quantization condition with respect to these gauge groups, therefore the Aharonov-Bohm phases of the charged particles going around the Dirac strings must come right. Here, I gave a description in terms of the $U(1)_{\text {broken }} \times U(1)_{\text {eff }}$ basis.

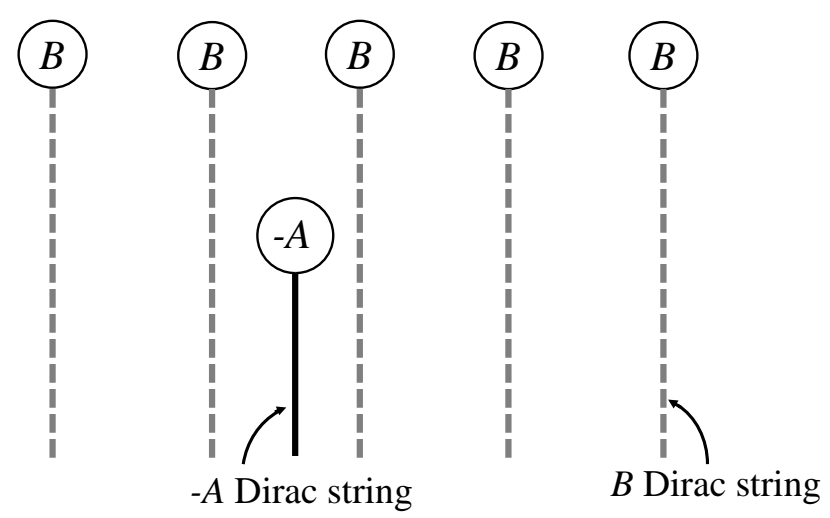

Figure 2: An anti-monopole of $U(1)_{A}$ and $Z$ monopoles of $U(1)_{B}$ in the unbroken phase of the $U(1)_{A} \times U(1)_{B}$ gauge theory.

Note that the physical magnetic flux in the Nielsen-Olesen flux tube has the unit flux. Since the Nielsen-Olesen flux tube is a solution of the gauge-Higgs system, the charge of the Higgs field determines the unit magnetic flux. The Higgs field has the charge with respect to the $U(1)_{\text {broken }}$ (combined with the gauge coupling constant) $g_{\text {eff }}\left(1+Z^{2}\right.$ ) (see Table 1). This leads to the quantization of the magnetic flux in the Nielsen-Olesen flux 


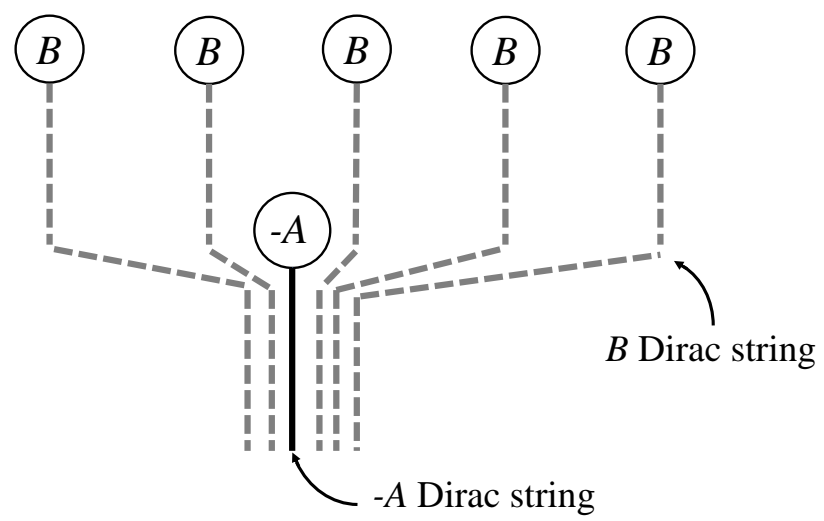

Figure 3: The monopoles in the broken phase of the gauge theory, but Dirac strings still in the basis of $U(1)_{A} \times U(1)_{B}$. While Dirac strings are not physical, the magnetic flux of the broken gauge group $U(1)_{\text {broken }}$ is physical. One can choose a gauge in which the Dirac string of $U(1)_{\text {broken }}$ is along the magnetic flux [35, 36].

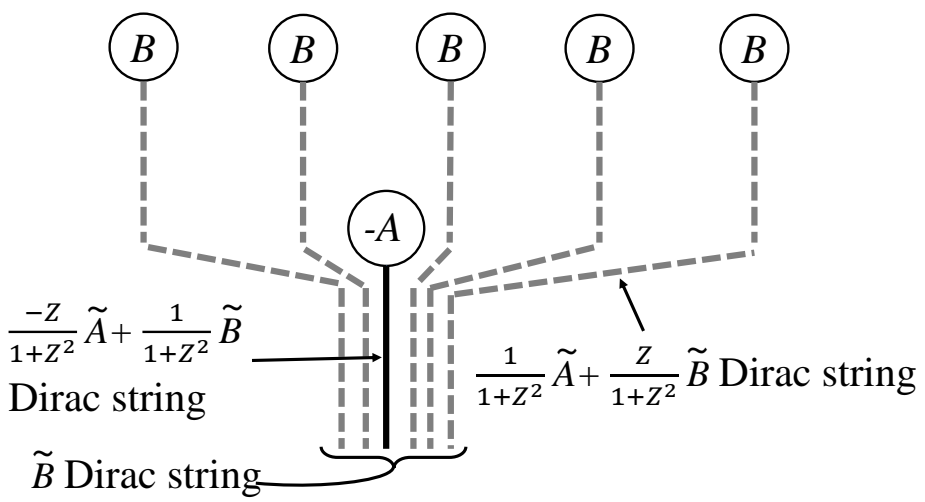

Figure 4: The same system as in Fig. 3, but in terms of the new basis $U(1)_{\text {broken }} \times U(1)_{\text {eff }}$. The Dirac string of $U(1)_{\text {broken }}$ is indicated by $\tilde{A}$ and the Dirac string of $U(1)_{\text {eff }}$ is indicated by $\tilde{B}$. The unit of the Dirac string of $U(1)_{\text {broken }}\left(U(1)_{\text {eff }}\right)$ is normalized so that that from the monopole with unit magnetic charge with respect to $U(1)_{\text {broken }}\left(U(1)_{\text {eff }}\right)$ is one. 


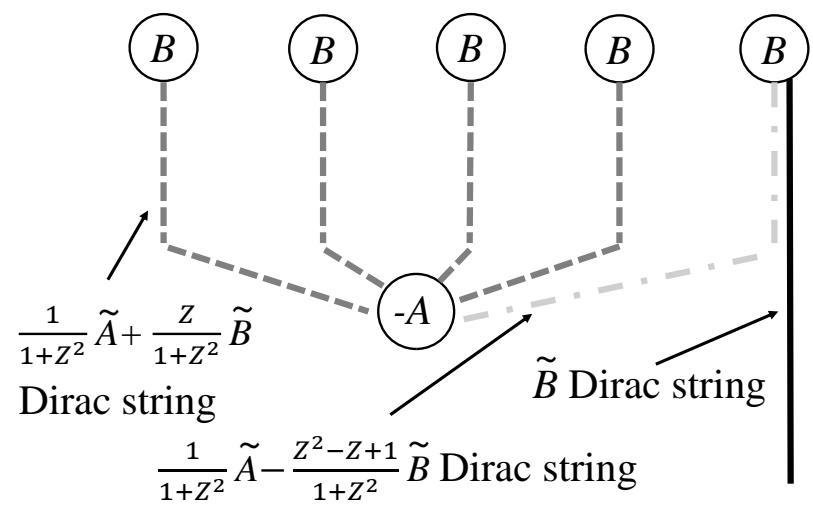

Figure 5: The same system as in Fig. 4, but in a different gauge. A gauge transformation in $U(1)_{\text {eff }}$ can move around one unit of the Dirac string of $U(1)_{\text {eff. }}$.

tube as

$$
\frac{2 \pi n}{g_{\mathrm{eff}}\left(1+Z^{2}\right)} \quad n \text { : integer . }
$$

Here, the integer $n$ is the vorticity ${ }^{17}$

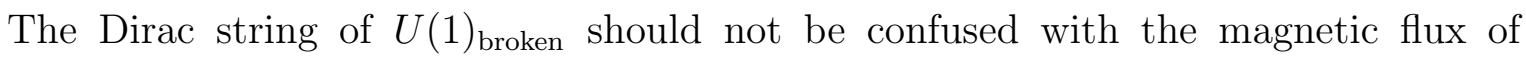
$U(1)_{\text {broken }}$ inside the flux tube [35]. The magnetic flux is physical and there is no problem in particles probing it through the Aharonov-Bohm effect. One can choose a gauge in which the Dirac string of $U(1)_{\text {broken }}$ can be placed along the magnetic flux by a choice of gauge [36].

The existence of the gauge field of $U(1)_{\text {broken }}$ and the charges with respect to it was crucial for making the Dirac strings from the monopoles of $U(1)_{A}$ and $U(1)_{B}$ with the fractional magnetic charges with respect to the $U(1)_{\text {eff }}$ gauge group unobservable. The gauge field of $U(1)_{\text {broken }}$ is a new physics beyond $\mathrm{EFT}_{\text {eff }}$. While the massive gauge bosons of $U(1)_{\text {broken }}$ do not appear as particle states at the energy scale below $m_{V}$, their existence is required so that the Dirac strings not to be probed through the Aharonov-Bohm effect. Therefore, the size $L_{m}$ of the monopole of $U(1)_{\text {eff }}$ is the scale where the description by $\mathrm{EFT}_{\text {eff }}$ breaks down. Thus I identify the size of the monopole of $U(1)_{\text {eff }}$ with the UV

\footnotetext{
${ }^{17}$ For a review of Nielsen-Olesen flux tube (vortex) and its topological property, see for example 34 .
} 
cut-off scale of $\mathrm{EFT}_{\text {eff }}{ }^{18}$

$$
\Lambda_{\mathrm{eff}} \sim \frac{1}{L_{m}} \sim \frac{g v}{\sqrt{Z}} .
$$

Notice that this UV cut-off scale $\Lambda_{\text {eff }}$ is much smaller than the mass $m_{V}=g \sqrt{1+Z^{2}} v$ (3.8) of the massive gauge field when $Z \gg 1$.

With the identification of the UV cut-off scale in (3.15), the mass of the monopole of $U(1)_{\text {eff }} 3.12$ coincides with the general expectation for the mass of the Dirac monopole in EFT given in 2.16):

$$
m_{m 0} \sim \frac{\Lambda_{\mathrm{eff}}}{g_{\mathrm{eff}}^{2}}
$$

Indeed, it is unnatural if the mass of the monopole depends on the detail of the UV theory.

Now I show that the magnetic WGC, in particular the bound on the UV cut-off scale (2.17) is satisfied in $\mathrm{EFT}_{\text {eff }}$, provided additional assumptions, which I make explicit below, hold. It is natural to assume that the vacuum expectation value of the Higgs field is below the UV cut-off scale of $\mathrm{EFT}_{\mathrm{UV}}, v<\Lambda \lesssim g M_{P} / \sqrt{2}$. Then, it follows that

$$
\begin{aligned}
\Lambda_{\mathrm{eff}} & \sim \frac{1}{L_{m}} \sim \frac{g v}{\sqrt{Z}} \\
& \lesssim \frac{g^{2} M_{P}}{\sqrt{2 Z}}=\left(\frac{g}{\sqrt{2 Z} \sin \gamma}\right) g_{\mathrm{eff}} M_{P}=\frac{g Z}{\sqrt{2 Z} \cos \gamma} g_{\mathrm{eff}} M_{P} \\
& \lesssim \frac{1}{\sqrt{Z}} g_{\mathrm{eff}} M_{P} \lesssim g_{\mathrm{eff}} M_{P},
\end{aligned}
$$

i.e. the bound on the UV cut-off scale 2.17) associated with $U(1)_{\text {eff }}$ gauge theory is (safely) satisfied. In deriving (3.17), I used $\sqrt{2} \cos \gamma \geq 1$ for $Z \geq 1$, and I assumed $g Z \lesssim 1$ so that the perturbative description of $\mathrm{EFT}_{\mathrm{UV}}$ is valid (recall that the Higgs field had charge $(Z, 1)$ in the original charge basis).

The two extra assumptions I made to show (3.17) were as follows: ${ }^{19}$

$$
\text { Assumption 1: } v<\Lambda \text {. }
$$

$$
\text { Assumption } 2: \quad g Z \lesssim 1 \text {. }
$$

To analyze the assumption 1, I first assume a quadratic potential for the Higgs field:

$$
V_{H}(H)=-\frac{\mu^{2}}{2}|H|^{2}+\frac{\lambda}{4 !}|H|^{4} .
$$

\footnotetext{
${ }^{18}$ In the original article [1, the WGC with respect to the $U(1)_{A} \times U(1)_{B}$ gauge theory and the WGC with respect to the $U(1)_{\text {eff }}$ gauge theory were not clearly distinguished. At $\Lambda_{\text {eff }}$ which is the UV cutoff scale for the $U(1)_{\text {eff }}$ gauge theory, the fractional magnetic charge with respect to the $U(1)_{\text {eff }}$ gauge group makes the $U(1)_{\text {eff }}$ gauge theory becomes theoretically inconsistent, and the new degrees of freedom associated with the $U(1)_{A} \times U(1)_{B}$ gauge theory manifest themselves through the Aharonov-Bohm effect.

${ }^{19}$ These assumptions were also made in the analysis by Saraswat [1].
} 
Then, the vacuum expectation value $v$ is given by

$$
v=\sqrt{\frac{6}{\lambda}} \mu .
$$

Here, I chose the gauge so that the vacuum expectation value $v$ becomes real. I already assumed that $m_{H}=\sqrt{2} \mu<\Lambda$ in (3.1) so that the creation of Higgs particles can be described within the $\mathrm{EFT}_{\mathrm{UV}}$. Therefore, in order to achieve $v \gg \Lambda$, a very small parameter $\lambda \ll 1$ is required. Thus in order to remove the assumption 1 , one need to introduce a tiny coupling which in view of naturalness requires an explanation. Also from naturalness, (3.20 is the most relevant part of the potential for the Higgs field, and the above estimate of the expectation value should not change significantly by introducing higher order interactions.

The assumption 2 is a requirement that $\mathrm{EFT}_{\mathrm{UV}}$ is in the perturbative regime. It would be difficult to make any quantitative conclusion without this assumption.

Now, I examine whether the electric WGC bound 2.12 is respected by the $\psi_{B}$ particles. The charge $\tilde{q}_{B}$ of the $\psi_{B}$ particles with respect to the gauge group $U(1)_{\text {eff }}$ in my convention is $\tilde{q}_{B}=Z$, see Table 1 . From 3.9 it follows that

$$
\frac{g_{\mathrm{eff}} \tilde{q}_{B} M_{P}}{m_{B}}=\frac{g \cos \gamma M_{P}}{m_{B}} \gtrsim \frac{\sqrt{2} \Lambda \cos \gamma}{m_{B}} \gtrsim 1
$$

where the model assumptions for EFT $_{\mathrm{UV}}$, i.e. 3.1 and 3.5 and $\sqrt{2} \cos \gamma \geq 1$ for $Z \geq 1$ have been used. Thus $\psi_{B}$ particles satisfy the electric WGC bound 2.12). In fact, one can show more generally that the weak electric WGC will not be violated by a spontaneous gauge symmetry breaking. This is explained in Appendix A. However, the strong electric WGC can be violated in EFT $_{\text {eff }}$ as shown below.

As for the $\psi_{A}$ particles, their charge with respect to $U(1)_{\text {eff }}$ is one in my convention. When $m_{A}>\Lambda_{\text {eff }}$ which is the case under study, $\psi_{A}$ may violate the electric WGC bound with respect to $U(1)$ eff:

$$
\frac{g_{\mathrm{eff}} M_{P}}{m_{A}}<\frac{g \sin \gamma M_{P}}{\Lambda_{\mathrm{eff}}}=g M_{P} \sin \gamma \frac{\sqrt{Z}}{g v}=\frac{M_{P}}{v} \sqrt{Z} \sin \gamma
$$

where 3.15 has been used. The vacuum expectation value of the Higgs field $v$ is an input parameter of $\mathrm{EFT}_{\mathrm{UV}}$ which controls the gauge symmetry breaking scale. For a given $v$, the right hand side of 3.23 can be made arbitrary small by taking $Z$ large. Therefore, $\psi_{A}$ particles may violate the electric WGC bound, depending on the choice of the parameter $Z$. 
When $m_{A}<m_{B}$, the lightest particle charged with respect to $U(1)_{\text {eff }}$ is $\psi_{A}$. Therefore, when $\psi_{A}$ particles do not satisfy the electric WGC bound (2.12), the strong version of the electric WGC is violated in $\mathrm{EFT}_{\text {eff }}$, while it was satisfied in $\mathrm{EFT}_{\mathrm{UV}}$. This is a notable feature of the Saraswat model [1].

Case 2: $\psi_{A}$ is known, $\psi_{B}$ is unknown to the low energy observers associated with $\mathrm{EFT}_{\text {eff }}$

As discussed in case 1 above, $\psi_{A}$ particles are not guaranteed to satisfy the electric WGC bound with respect to the $U(1)_{\text {eff }}$ gauge group. On the other hand, the field $\psi_{B}$ gives rise to particles which satisfy the electric WGC bound (2.12). Therefore, if the low energy observers associated with $\mathrm{EFT}_{\text {eff }}$ do not know the existence of $\psi_{B}$ particles, when $\psi_{A}$ particles do not satisfy the WGC bound with respect to the $U(1)_{\text {eff }}$ gauge group, the electric WGC may appear to be violated for the low energy observers associated with $\mathrm{EFT}_{\text {eff }}$.

Case 3: $\psi_{A}$ is unknown, $\psi_{B}$ is known to to the low energy observers associated with EFT $_{\text {eff }}$

In this case, the charged particles known to the low energy observers associated with the $\mathrm{EFT}_{\mathrm{EFT}}$ are only $\psi_{B}$ particles. Then, in my convention, the gauge coupling constant is normalized as

$$
g_{\mathrm{eff} 2}=g \cos \gamma=g_{\mathrm{eff}} \cot \gamma .
$$

Here, I have introduced a new notation $g_{\text {eff2 }}$ to distinguish it from the coupling constant $g_{\text {eff }}$ which was used in the case 1. Then, the low energy observers associated with $\mathrm{EFT}_{\text {eff }}$ predict that the bound on the UV cut-off scale $\Lambda_{\text {eff }}$ of $\mathrm{EFT}_{\text {eff }}$ is given by

$$
\Lambda_{\text {eff }} \lesssim g_{\text {eff2 }} M_{P}=g_{\text {eff }} \cot \gamma M_{P} .
$$

Since $\cot \gamma \geq 1$ for $Z \geq 1$, comparing with (3.17) it is clear that (3.25) is satisfied.

Case 4: Neither $\psi_{A}$ nor $\psi_{B}$ are known to to the low energy observers associated with $\mathbf{E F T}_{\text {eff }}$

In this case, there is no charged particle known to the low energy observers associated with $\mathrm{EFT}_{\text {eff. }}$ Then, the electric WGC appears to be violated, assuming that the low energy observers know the existence of the gauge field through some non-minimal coupling to the gauge field. Otherwise, the low energy observers do not recognize EFT as gauge theory, 
and there is no room to discuss the WGC from the beginning. Without a charged particle minimally coupled to the gauge field known, the low energy observers do not know what could be the smallest magnetic charge of the monopole. The lack of the knowledge can be stated as the bound. Without any information of the smallest charge, the low energy observers can say $g q_{0} \lesssim 1$ is still not forbidden, assuming that the EFT is in perturbative regime, where $q_{0}$ is the possible smallest charge. In the current convention $q_{0}=1$, and this gives a bound $g \lesssim 1$. It follows that the mass of the lightest monopole is bounded as $m_{m 0} \gtrsim \Lambda$ as in (2.16), which gives the bound on the UV cut-off scale $\Lambda \lesssim M_{P}$. However, one usually assumes that the UV cut-off scale of EFTs is below the Planck scale. Thus the WGC does not add more constraint on the UV cut-off scale of the EFT under consideration than what one usually already assumes in EFTs.

\subsection{Implications to the bottom-up model buildings}

Below I draw lessons from the analysis of the Saraswat model for general bottom-up model buildings. In Sec. 2.7, after the examination of the BH discharge processes, I assumed that the actual discharge process need not be efficient. Then, it followed that the charged particles which satisfy the electric WGC need not have mass below the UV cut-off scale of EFTs. If this is the case, the constraining power of the electric WGC on EFTs is quite limited. In fact, usually in the model building, one does not worry too much about stable heavy particles whose mass is above the UV cut-off scale of the EFT. This is because their quantum effects are renormalized into the parameters of the low energy EFT and cannot be separately measurable in the low energy experiments [37, 38]. Their classical effects as sources can be taken into account, but if these stable heavy particles are not around, there is no need to include them. What this means in the bottom-up approach to model buildings is that if one has a model which does not satisfy the electric WGC, one can simply assume that such particle exists with mass above the UV cut-off scale of the EFT to make this model satisfy the electric WGC. Theoretically, these stable heavy particles can be included in EFT as HPET, but practically, as long as one does not have such particles around, there would not be much need for studying processes involving the stable heavy particles.

However, the bound on the UV cut-off scale 2.17) derived from the magnetic WGC still gives an interesting constraint. To state the magnetic WGC, the low energy observers associated with the EFT need to know the existence of at least one charged particle state. With respect to the known charge, the low energy observers can give physically meaningful normalization of the gauge coupling constant, which can be used to unambiguously state 
the magnetic WGC and the bound on the UV cut-off scale of the EFT. In this article, I use the normalization with which the smallest charge known to the low energy observers is one. With this convention, the bound on the UV cut-off scale is stated as in 2.17), which gives a non-trivial constraint on EFTs from the WGC. As I have stressed, the knowledge of the low energy observers could be limited, and as a consequence the bound on the UV cut-off scale could be looser than the true bound derived from the magnetic WGC assuming it holds. However, a bound looser than the true bound is not wrong, it is just less constraining. It still gives the best bound for the given knowledge of the smallest charge. The case 3 was an example of the looser bound on the UV cut-off scale predicted by the low energy observers.

After the general discussions above, now I examine the implications of the limitation of the low energy observers associated with EFTs further in a specific example: The extra-natural inflation model based on the Saraswat model will be studied.

\section{Implications to extra-natural inflation models}

One of the original motivations of the WGC was to explain why it appeared difficult to realize extra-natural inflation in string theory [5] compatible with the observations such as [39]. In natural inflation models, the inflaton need to make a super-Planckian field excursion in order to be consistent with the observations of the spectral tilt and the bound on the tensor-to-scalar ratio, assuming the number of e-folds to be $50-60$. The extra-natural inflation realizes the super-Planckian field excursion at the cost of tiny gauge coupling constant (typically $\mathcal{O}\left(10^{-3}\right)$ or less [5, 40]) and the non-local nature of Wilson loops winding the extra dimension. The magnetic WGC, or more explicitly the bound on the UV cut-off scale (2.17), poses an obstruction for realizing such tiny gauge coupling constant with a required UV cut-off scale.

The Saraswat model discussed in the previous section achieves a tiny gauge coupling constant $g_{\text {eff }}$ from the large hierarchy between charges, $Z \gg 1$. I examine whether this mechanism can help achieving the super-Planckian inflaton excursion. It is straightforward to generalize the Saraswat model discussed in the previous section to 5D. Then, the 5 -th component of the 5D gauge fields $A_{\mu}$ and $B_{\mu}$, with $\mu$ now runs from $0, \cdots, 3$ and 5 , gives rise to axionic scalar fields in $4 \mathrm{D}$ with periodicity $2 \pi f{ }^{20}$ where the axion decay constant $f$ is given by

$$
f=\frac{1}{2 \pi g L_{5}}
$$

\footnotetext{
${ }^{20}$ The periodicity of the axion fields is a consequence of the gauge symmetry, and it remains even after the gauge field acquires a mass [41].
} 
Here, $L_{5}$ is the radius of the compact 5 -th direction, which I assume to be a circle. The $4 \mathrm{D}$ gauge coupling constant $g$ is related to the $5 \mathrm{D}$ gauge coupling constant $g_{5}$ as $g=g_{5} / \sqrt{2 \pi L_{5}}$. I require that the compactification scale is below the UV cut-off scale $\Lambda$ of the $\mathrm{EFT}_{\mathrm{UV}}$ :

$$
\frac{1}{L_{5}} \lesssim \Lambda \lesssim \frac{g M_{P}}{\sqrt{2}}
$$

where I assumed that the bound on the UV cut-off scale 3.5 holds. Putting (3.26) into (3.27), a bound on the axion decay constant $f$ is obtained:

$$
f \lesssim M_{P}
$$

The constraint on the axion decay constant like (3.28) poses an obstruction for achieving a super-Planckian field excursion of inflaton in the extra-natural inflation model with a single gauge field. However, in the Saraswat model, there are two gauge fields, and the inflaton trajectory need not be parallel to the periodic direction. As a consequence, the inflaton field excursion need not be restricted by the period $2 \pi f$. Indeed, the spontaneous gauge symmetry breaking adds a potential which tilt the inflaton trajectory from the periodic direction. I denote the canonically normalized fields which arise from the zeromodes of the 5-th dimensional component of the gauge fields $A_{5}$ and $B_{5}$ as $A$ and $B$, respectively. Fields with charge vectors $(1,0)$ and $(0,1)$ whose masses are light compared with the compactification scale $L_{5}$ contribute to the potential for $A$ and $B$ (see for example [42] for the details of the calculations). Since I assumed $1 / L_{5}<\Lambda$ as in (3.27), following the discussions in Sect. 2.5, these light charged fields give rise to particles which satisfy the electric WGC bound 2.12 with respect to the gauge group $U(1)_{A} \times U(1)_{B}$. Thus in extra-natural inflation models, the particles which satisfy the electric WGC bound are included in order to generate a suitable inflaton potential. The potential for the field $A$ and the field $B$ is given as

$$
\begin{aligned}
V(\tilde{A}, \tilde{B}) & \sim C_{1} \cos \left(\frac{A}{f}\right)+C_{2} \cos \left(\frac{B}{f}\right)+\frac{1}{2}(g Z v A+g v B)^{2} \\
& \sim C_{1} \cos \left(\frac{\cos \gamma \tilde{A}-\sin \gamma \tilde{B}}{f}\right)+C_{2} \cos \left(\frac{\sin \gamma \tilde{A}+\cos \gamma \tilde{B}}{f}\right)+\frac{m_{V}^{2}}{2} \tilde{A}^{2} .
\end{aligned}
$$

Here, $\tilde{A}$ and $\tilde{B}$ are defined using the linear transformation as in $(3.6)$ :

$$
\left(\begin{array}{c}
\tilde{A} \\
\tilde{B}
\end{array}\right)=\left(\begin{array}{cc}
\cos \gamma & \sin \gamma \\
-\sin \gamma & \cos \gamma
\end{array}\right)\left(\begin{array}{c}
A \\
B
\end{array}\right),
$$

where

$$
\cos \gamma:=\frac{Z}{\sqrt{1+Z^{2}}}, \quad \sin \gamma:=\frac{1}{\sqrt{1+Z^{2}}}
$$


In (3.29), $m_{V}=g \sqrt{1+Z^{2}} v$ as in (3.8), and $C_{1}$ and $C_{2}$ depend on the number of the light fields with charge vector $(1,0)$ and $(0,1)$, respectively. The field $\tilde{B}$ is to be identified with the inflaton. Then, to achieve the large axion decay constant, I assume $C_{1} \gg C_{2}$ so that the second sinusoidal potential is suppressed. ${ }^{21}$ I further assume $C_{1} / f \ll m_{V}^{2} \tilde{A}$ so that the field $\tilde{A}$ settles down to its minimum $\tilde{A} \sim 0$ first. Under these assumptions, I obtain the effective potential for the field $\tilde{B}$ as

$$
V_{\text {eff }}(\tilde{B}) \sim C_{1} \cos \left(\frac{-\tilde{B}}{f_{\text {eff }}}\right),
$$

where

$$
f_{\text {eff }}=\frac{f}{\sin \gamma} \lesssim \frac{M_{P}}{\sin \gamma}
$$

Therefore, the bound on the effective axion decay constant $f_{\text {eff }}$ is much milder than that for $f(3.28)$ if $Z \gg 1$, and $f_{\text {eff }}$ can be super-Planckian if $Z$ is large enough. This enhancement of the effective axion decay constant by tilting of the inflaton trajectory with respect to the periodic direction is the same mechanism employed in axion-monodromy inflation models [44, 45, 46, 47, 48, 49]. However, in the current model, the monodromy (going round in periodic direction more than once) itself is not used.

Thus the extra-natural inflation model based on the Saraswat model may achieve super-Planckian inflaton travel without violating the WGC. However, the large hierarchy between charges $Z \gg 1$ required to achieve the super-Planckian inflaton excursion is a potential obstruction for embedding this model to string theory [31, 50, 51, 52].

It is important to notice that the extra-dimensional aspect of this model can only be described by the high energy theory $\mathrm{EFT}_{\mathrm{UV}}$, not $\mathrm{EFT}_{\text {eff }}$. This is because when the super-Planckian effective axion decay constant $f_{\text {eff }} \gtrsim M_{P}$ is achieved, from (3.17)

$$
\Lambda_{\mathrm{eff}} \lesssim g_{\mathrm{eff}} M_{P} \lesssim g_{\mathrm{eff}} f_{\mathrm{eff}} \sim \frac{1}{L_{5}}
$$

Here, I have used

$$
g_{\mathrm{eff}} f_{\mathrm{eff}}=g f=\frac{1}{2 \pi L_{5}},
$$

which follows from $(3.26),(3.33)$ and $(3.9)$. Thus the low energy observers associated with $\mathrm{EFT}_{\text {eff }}$ cannot resolve the extra dimension. This means that while $\mathrm{EFT}_{\text {eff }}$ achieves a tiny gauge coupling constant, it cannot be used as a model of extra-natural inflation. It is a genuinely 4D model and cannot explain the super-Planckian axion decay constant. The

\footnotetext{
${ }^{21}$ The periodic modulation by the second sinusoidal potential is subject to the observational constraints [43.
} 
$5 \mathrm{D}$ model $\mathrm{EFT}_{\mathrm{UV}}$, which satisfies (3.27), is required as a model of extra-natural inflation. Accordingly, the relevant WGC relevant for constraining the inflaton field range is that on $\mathrm{EFT}_{\mathrm{UV}}$, not the WGC on $\mathrm{EFT}_{\mathrm{UV}}$. In $\mathrm{EFT}_{\mathrm{UV}}$ the gauge group is $U(1)_{A} \times U(1)_{B}$ with the gauge coupling constant $g$. Thus it is more appropriate to view the extra-natural inflation model based on the 5D version of the Saraswat model as a model achieving the super-Planckian inflaton travel by the tilt of the inflaton trajectory from the periodic direction, rather than to view it as an extra-natural inflation model with a tiny gauge coupling constant $g_{\text {eff }}$.

\section{Summary and discussions}

In this article, I examined the WGC from the low energy observers' perspective, to address the issue of to what extent the WGC actual constrains EFTs. For this purpose, I introduced idealized low energy observers for a given EFT, who have full access to particles whose mass is below the UV cut-off scale $\Lambda$ of the EFT. However, I did not assume that all the stable particles whose mass is above $\Lambda$ are known to the low energy observers. This is because creations of particles heavier than $\Lambda$ occur only at energy higher than $\Lambda$ and those are not under control of the EFT. Since I considered idealized low energy observers, this limitation of the knowledge of the low energy observers is fundamental and it is not due to experimental practicalities. An immediate consequence of the limitation of the knowledge of the low energy observers associated with Abelian gauge EFTs was that the low energy observers can never be sure what is the smallest charge in Nature. It also follows that they are not sure about the smallest magnetic charge neither. What the low energy observers can do was to constrain possible magnetic charges based on the knowledge of the smallest electric charge via the Dirac quantization condition. The existence of a charged particle was necessary to give a physically meaningful normalization of the gauge coupling constant, and it was crucial for unambiguously stating the magnetic WGC.

I gave two main reasons for considering stable heavy particles whose mass is above the UV cut-off scale of the EFT under consideration. The first reason was that it is of practical importance to ask if the low energy IR EFT satisfies the WGC when it is derived from UV EFT which satisfies the WGC. The UV EFT may have charged particles whose mass is below its UV cut-off scale, but their mass can be above the UV cut-off scale of the IR EFT. If this is the case, it is easy to construct a model in which in the UV EFT the WGC is satisfied but in the IR EFT the WGC appears to be violated, if the low energy observers associated with the IR EFT know nothing about the stable heavy particles 
whose mass is above the UV cut-off scale of the IR EFT. When the WGC appears to be violated, it does not constrain the IR EFT. The WGC gives non-trivial constraints only when the low energy observers associated with the IR EFT know all or some of the stable heavy particles whose mass is above the UV cut-off scale of the IR EFT. The examination of the $\mathrm{BH}$ discharge arguments in Sec. 2.7 enhances this reasoning for considering stable particles above the UV cut-off scale of the EFT under consideration. The charged particles responsible for the discharge of BHs can have non-zero mass. This means that one can integrate out degrees of freedom above the mass of the lightest charged particle which satisfies the electric WGC bound (2.12) to obtain an EFT. Since there is no charged particle in the EFT thus obtained, the electric WGC appears to be violated unless one takes into account the charged particles whose mass is above the UV cut-off scale of the EFT. The second reason for taking into account stable heavy particles was that once the magnetic WGC, or more precisely, the bound on the UV cut-off scale (2.17) is assumed, charged particles whose mass is below the UV cut-off scale automatically satisfy the electric WGC bound, as explained in Sec. 2.5. Therefore, the electric WGC becomes a non-trivial question only when there is no charged particle below the UV cut-off scale.

I illustrated the above points taking the Saraswat model [1] as an example. In the Saraswat model, strong electric WGC can be violated at low energy, while it is respected at high energy. When some of the charged particles whose mass is above the UV cut-off scale of the low energy EFT are not known to the low energy observers, the weak electric WGC may also appear to be violated for the low energy observers. In this article, I also showed that the magnetic WGC is not violated at low energy. To show this result, it was important to correctly identify the UV cut-off scale of the low energy theory. This was done by a detailed analysis of the internal structure of the monopole of $U(1)_{\text {eff }}$. the fractional magnetic charges of the constituent monopoles with respect to $U(1)_{\text {eff }}$ reveals

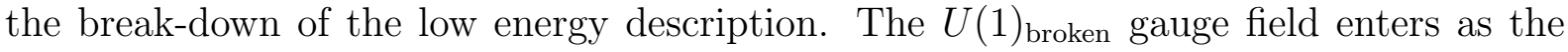
new physics which replaces the low energy description. While the massive gauge bosons still do not appear as particle states at the energy scale corresponding to the size of the monopole of $U(1)_{\text {eff }}$, their existence is required to keep the Dirac strings unphysical.

The limitation of the knowledge of the low energy observers on the existence of charged particles whose mass is above the UV cut-off scale of the EFT leads to the limitation of the constraining power of the WGC on bottom-up model buildings using EFTs. Regarding the electric WGC, one can freely construct a model based on an EFT, and if the model does not contain a particle which satisfies the electric WGC bound (2.12), one can simply assume the existence of particles which satisfy the electric WGC bound above the UV cut-off scale. One can further assume that they were not created with observable density. 
Since below the UV cut-off scale the quantum effects of the stable heavy particles are renormalized into parameters of the EFT, the additional assumption does not practically modify the model. On the other hand, when a charged particle is known to the low energy observers, one can give a physical definition of the gauge coupling constant to state the magnetic WGC which in turn puts a bound on the UV cut-off scale of the EFT. Due to the limitation of the knowledge of the low energy observers regarding the charges of particles, the bound on the UV cut-off scale can be looser than the one obtained from the true smallest charge assuming the magnetic WGC to hold. Nevertheless, the looser bound is not violated by the true bound, it is just less constraining. But the looser bound is still the best bound for the given knowledge.

The lessons from the analysis of the Saraswat model for general bottom-up model buildings based on EFTs were discussed in Sec. 3.2. The implications to extra-natural inflation models were studied in some detail. The extra-natural inflation model based on the Saraswat model provides an example in which WGC alone does not forbid superPlanckian inflaton excursion. However, in this model, the large hierarchy between charges required to achieve the super-Planckian inflaton excursion can be a potential obstacle for realizing this models in string theory. At the moment, there is no universal formula for the bound on the hierarchy between charges which applies to a large class of EFTs. It will be interesting to look for a criterion for separating out EFTs which cannot be realized in string theory due to the large hierarchy between charges.

As I discussed in Sec. 2.7, the requirement that BH should be able to release more charges than mass in Planck unit does not constrain the mass of the charged particles, under the assumption I made that BHs only need to be kinematically allowed to discharge, but the actual physical process for the discharge need not be efficient. In particular, the mass of the charged particle which satisfy the electric WGC bound can be above the UV cut-off scale of the EFT under consideration. The lack of necessity of the efficient BH discharge process made me suspect that the $\mathrm{BH}$ discharge process may not be relevant for realizing the low energy world satisfying the WGC. I suspect that there is a more fundamental principle which prevents the WGC to be violated. A possible clue for identifying such hypothetical fundamental principle may be that for the magnetic WGC, there is a physically independent argument in support for the bound on the UV cut-off scale: The monopole with the smallest magnetic charge should not be a $\mathrm{BH}$ [3]. Indeed, it is strange if the monopole with the smallest magnetic charge is already a $\mathrm{BH}$, given a widely accepted belief that the Bekenstein-Hawking entropy has a microscopic origin. I suspect that this argument may be more directly related to the fundamental principle behind the 
WGC, which is likely to be some sort of entropy bound ${ }^{22}$ However, heuristic arguments based on an entropy bound should be taken with caution, in light of the quantitative understanding of the Bekenstein bound [55]. In the context of giving more emphasis on the magnetic WGC and the bound on the UV cut-off scale, I find it indicative that once the magnetic WGC, in particular the bound on the UV cut-off scale on an EFT is assumed, the electric WGC automatically follows if there exists a charged particle whose mass is below the UV cut-off scale. This observation may provide a step for deriving the electric WGC from the hypothetical entropy bound.

\section{Acknowledgments}

I would like to thank Yoji Koyama for collaboration at the early stage of this work as well as careful reading of the manuscript. I would also like to thank Cheng-Yang Lee for useful discussions. This work is supported in part by the Science and Engineering Research Board, Department of Science and Technology, Government of India under the grant No. EMR/2015/002471.

\section{A The weak electric WGC is not violated by spon- taneous gauge symmetry breakings}

In this appendix, I show that starting from gauged EFT with product of $U(1)$ gauge groups, the weak electric WGC will never be violated by spontaneous gauge symmetry breaking. For this purpose, I need to recall the result of [18]. Let $\Pi_{\alpha=1}^{N} U(1)_{\alpha}$ be the gauge group of some EFT. In this appendix, I use a normalization for the gauge coupling and the charge different from the main body: The product of the charge and the gauge coupling $g_{\alpha} q_{\alpha}$ (no sum in $\alpha$ ) in the notation of the main body is simply denoted as $q_{\alpha}$ in this appendix.

Consider a $\mathrm{BH}$ with charge $\vec{Q}$ and mass $M$. I define charge-to-mass vector $\vec{Z}$ as

$$
\vec{Z}=\frac{\vec{Q} M_{P}}{M} .
$$

Consider a decay of the BH to a final state consisting of $n_{i}$ particles of spices $i$ with charge vector $\vec{q}_{i}$ and mass $m_{i}$. The charge conservation implies

$$
\vec{Q}=\sum_{i} n_{i} \vec{q}_{i}
$$

\footnotetext{
${ }^{22}$ Related ideas have been pursued in [53, 54].
} 
The energy conservation implies

$$
M>\sum_{i} n_{i} m_{i}
$$

Let $\sigma_{i}:=n_{i} m_{i} / M$ be the spices $i$ fraction of the total final state mass. Then A.2 can be rewritten as

$$
\vec{Z}=\sum_{i} \sigma_{i} \vec{z}_{i}
$$

where

$$
\vec{z}_{i}:=\frac{\vec{q}_{i} M_{P}}{m_{i}}
$$

while A.3 can be rewritten as

$$
1>\sum_{i} \sigma_{i}
$$

Thus the requirement that the $\mathrm{BH}$ is kinematically allowed to decay amounts to the condition that $\vec{Z}$ be a sub-unitary weighted average of $\vec{z}_{i}$. The geometric interpretation of this condition is that the $N$-dimensional unit ball $|\vec{Z}| \leq 1$ representing the BPS bound 2.24 is contained in the convex hull spanned by the vectors $\vec{z}_{i}$.

In this article, I focused on Coulomb force mediated by massless gauge fields. In this setting, a spontaneous gauge symmetry breaking amounts to projecting charge vectors into space spanned by charges of the remaining massless $U(1)$ 's ${ }^{23}$ Suppose that $p<N$ independent linear combinations of the $U(1)$ gauge fields become massive. I denote the projection $P$ to the subspace in the charge-to-mass vector space where charges for the massless gauge fields remain. Clearly, if the $N$-dimensional unit ball is contained in the original convex hull spanned by the vectors $\vec{z}_{i}$, the $(N-p)$-dimensional unit ball in the projected space is also contained in the convex hull spanned by the projected vectors $P \vec{z}_{i}$. This means that if one starts from the EFT which respects the weak electric WGC, the weak electric WGC will not be violated by the spontaneous gauge symmetry breaking.

\section{References}

[1] P. Saraswat, "Weak gravity conjecture and effective field theory," Phys. Rev. D95 no. 2, (2017) 025013, arXiv:1608.06951 [hep-th].

[2] C. Vafa, "The String landscape and the swampland," arXiv:hep-th/0509212 [hep-th].

\footnotetext{
${ }^{23}$ The charge of BHs associated with massive gauge fields may be probed using Aharonov-Bohm effect [56. Implication of this observation to the WGC is currently not clear to me, but it may be an interesting direction to investigate.
} 
[3] N. Arkani-Hamed, L. Motl, A. Nicolis, and C. Vafa, "The String landscape, black holes and gravity as the weakest force," JHEP 06 (2007) 060, arXiv:hep-th/0601001 [hep-th].

[4] T. Banks, M. Johnson, and A. Shomer, "A Note on Gauge Theories Coupled to Gravity," JHEP 09 (2006) 049, arXiv:hep-th/0606277 [hep-th].

[5] N. Arkani-Hamed, H.-C. Cheng, P. Creminelli, and L. Randall, "Extra natural inflation," Phys. Rev. Lett. 90 (2003) 221302, arXiv:hep-th/0301218 [hep-th].

[6] M. Neubert, "Heavy quark effective theory," Subnucl. Ser. 34 (1997) 98-165, arXiv:hep-ph/9610266 [hep-ph].

[7] A. V. Manohar and M. B. Wise, "Heavy quark physics," Camb. Monogr. Part. Phys. Nucl. Phys. Cosmol. 10 (2000) 1-191.

[8] A. G. Grozin, "Heavy quark effective theory," Springer Tracts Mod. Phys. 201 (2004) 1-213.

[9] A. H. Guth, "The Inflationary Universe: A Possible Solution to the Horizon and Flatness Problems," Phys. Rev. D23 (1981) 347-356.

[10] M. B. Einhorn and K. Sato, "Monopole Production in the Very Early Universe in a First Order Phase Transition," Nucl. Phys. B180 (1981) 385-404.

[11] A. D. Linde, "A New Inflationary Universe Scenario: A Possible Solution of the Horizon, Flatness, Homogeneity, Isotropy and Primordial Monopole Problems," Phys. Lett. 108B (1982) 389-393.

[12] H. Georgi and M. B. Wise, "Superflavor Symmetry for Heavy Particles," Phys. Lett. B243 (1990) 279-283.

[13] C. D. Carone, "Superflavor symmetry for heavy vector mesons and heavy quarks," Phys. Lett. B253 (1991) 408-410.

[14] N. Isgur and M. B. Wise, "Weak Decays of Heavy Mesons in the Static Quark Approximation," Phys. Lett. B232 (1989) 113-117.

[15] N. Isgur and M. B. Wise, "WEAK TRANSITION FORM-FACTORS BETWEEN HEAVY MESONS," Phys. Lett. B237 (1990) 527-530. 
[16] E. Eichten and B. R. Hill, "An Effective Field Theory for the Calculation of Matrix Elements Involving Heavy Quarks," Phys. Lett. B234 (1990) 511-516.

[17] H. Georgi, "An Effective Field Theory for Heavy Quarks at Low-energies," Phys. Lett. B240 (1990) 447-450.

[18] C. Cheung and G. N. Remmen, "Naturalness and the Weak Gravity Conjecture," Phys. Rev. Lett. 113 (2014) 051601, arXiv:1402.2287 [hep-ph].

[19] B. Heidenreich, M. Reece, and T. Rudelius, "Weak Gravity Strongly Constrains Large-Field Axion Inflation," JHEP 12 (2015) 108, arXiv:1506.03447 [hep-th].

[20] B. Heidenreich, M. Reece, and T. Rudelius, "Sharpening the Weak Gravity Conjecture with Dimensional Reduction," JHEP 02 (2016) 140, arXiv:1509.06374 [hep-th].

[21] A. Hebecker, F. Rompineve, and A. Westphal, "Axion Monodromy and the Weak Gravity Conjecture," JHEP 04 (2016) 157, arXiv:1512.03768 [hep-th].

[22] B. Heidenreich, M. Reece, and T. Rudelius, "Evidence for a sublattice weak gravity conjecture," JHEP 08 (2017) 025, arXiv:1606.08437 [hep-th].

[23] M. Montero, G. Shiu, and P. Soler, "The Weak Gravity Conjecture in three dimensions," JHEP 10 (2016) 159, arXiv:1606.08438 [hep-th].

[24] G. W. Gibbons, "Vacuum Polarization and the Spontaneous Loss of Charge by Black Holes," Commun. Math. Phys. 44 (1975) 245-264.

[25] G. F. Giudice, "Naturally Speaking: The Naturalness Criterion and Physics at the LHC," arXiv:0801.2562 [hep-ph].

[26] T. Crisford, G. T. Horowitz, and J. E. Santos, "Testing the Weak Gravity - Cosmic Censorship Connection," arXiv:1709.07880 [hep-th].

[27] M. K. Parikh and F. Wilczek, "Hawking radiation as tunneling," Phys. Rev. Lett. 85 (2000) 5042-5045, arXiv:hep-th/9907001 [hep-th].

[28] E. Brezin and C. Itzykson, "Pair production in vacuum by an alternating field," Phys. Rev. D2 (1970) 1191-1199. 
[29] S. B. Giddings, "The Black hole information paradox," in Particles, strings and cosmology. Proceedings, 19th Johns Hopkins Workshop and 5th PASCOS

Interdisciplinary Symposium, Baltimore, USA, March 22-25, 1995, pp. 415-428. 1995. arXiv:hep-th/9508151 [hep-th].

[30] A. Hebecker, P. Mangat, F. Rompineve, and L. T. Witkowski, "Winding out of the Swamp: Evading the Weak Gravity Conjecture with F-term Winding Inflation?," Phys. Lett. B748 (2015) 455-462, arXiv:1503.07912 [hep-th].

[31] L. E. Ibanez and M. Montero, "A Note on the WGC, Effective Field Theory and Clockwork within String Theory," arXiv:1709.02392 [hep-th].

[32] H. B. Nielsen and P. Olesen, "Vortex Line Models for Dual Strings," Nucl. Phys. B61 (1973) 45-61.

[33] Y. Nambu, "Strings, Monopoles and Gauge Fields," Phys. Rev. D10 (1974) 4262.

[34] E. J. Weinberg, "Classical solutions in quantum field theories," Ann. Rev. Nucl. Part. Sci. 42 (1992) 177-210.

[35] M. Creutz, "The Higgs Mechanism and Quark Confinement," Phys. Rev. D10 (1974) 2696.

[36] G. Ripka, "Dual superconductor models of color confinement," Lect. Notes Phys. 639 (2004) pp.1-135, arXiv:hep-ph/0310102 [hep-ph].

[37] T. Appelquist and J. Carazzone, "Infrared Singularities and Massive Fields," Phys. Rev. D11 (1975) 2856.

[38] B. A. Ovrut and H. J. Schnitzer, "Decoupling Theorems for Effective Field Theories," Phys. Rev. D22 (1980) 2518.

[39] Planck Collaboration, P. A. R. Ade et al., "Planck 2015 results. XX. Constraints on inflation," Astron. Astrophys. 594 (2016) A20, arXiv:1502.02114 [astro-ph.CO].

[40] K. Furuuchi and J. M. S. Wu, " $U(1)_{B-L}$ extra-natural inflation with Standard Model on a brane," Phys. Lett. B729 (2014) 56-61, arXiv:1310.4646 [hep-ph].

[41] K. Furuuchi, "Excursions through KK modes," JCAP 1607 no. 07, (2016) 008, arXiv: 1512.04684 [hep-th]. 
[42] K. Furuuchi and Y. Koyama, "Large field inflation models from higher-dimensional gauge theories," JCAP 1502 no. 02, (2015) 031, arXiv:1407.1951 [hep-th].

[43] R. Flauger, L. McAllister, E. Pajer, A. Westphal, and G. Xu, "Oscillations in the CMB from Axion Monodromy Inflation," JCAP 1006 (2010) 009, arXiv:0907.2916 [hep-th].

[44] J. E. Kim, H. P. Nilles, and M. Peloso, "Completing natural inflation," JCAP 0501 (2005) 005, arXiv:hep-ph/0409138 [hep-ph].

[45] E. Silverstein and A. Westphal, "Monodromy in the CMB: Gravity Waves and String Inflation," Phys. Rev. D78 (2008) 106003, arXiv:0803.3085 [hep-th].

[46] L. McAllister, E. Silverstein, and A. Westphal, "Gravity Waves and Linear Inflation from Axion Monodromy," Phys. Rev. D82 (2010) 046003, arXiv:0808.0706 [hep-th].

[47] M. Berg, E. Pajer, and S. Sjors, "Dante's Inferno," Phys. Rev. D81 (2010) 103535, arXiv:0912.1341 [hep-th].

[48] R. Kappl, S. Krippendorf, and H. P. Nilles, "Aligned Natural Inflation: Monodromies of two Axions," Phys. Lett. B737 (2014) 124-128, arXiv:1404.7127 [hep-th].

[49] I. Ben-Dayan, F. G. Pedro, and A. Westphal, "Hierarchical Axion Inflation," Phys. Rev. Lett. 113 (2014) 261301, arXiv:1404.7773 [hep-th].

[50] G. Shiu, P. Soler, and F. Ye, "Milli-Charged Dark Matter in Quantum Gravity and String Theory," Phys. Rev. Lett. 110 no. 24, (2013) 241304, arXiv:1302.5471 [hep-th].

[51] L. McAllister, P. Schwaller, G. Servant, J. Stout, and A. Westphal, "Runaway Relaxion Monodromy," arXiv:1610.05320 [hep-th].

[52] A. Hebecker, P. Henkenjohann, and L. T. Witkowski, "Flat Monodromies and a Moduli Space Size Conjecture," JHEP 12 (2017) 033, arXiv:1708.06761 [hep-th].

[53] A. de la Fuente, P. Saraswat, and R. Sundrum, "Natural Inflation and Quantum Gravity," Phys. Rev. Lett. 114 no. 15, (2015) 151303, arXiv:1412.3457 [hep-th]. 
[54] Z. Fisher and C. J. Mogni, "A Semiclassical, Entropic Proof of a Weak Gravity Conjecture," arXiv:1706.08257 [hep-th].

[55] H. Casini, "Relative entropy and the Bekenstein bound," Class. Quant. Grav. 25 (2008) 205021, arXiv:0804.2182 [hep-th].

[56] J. Preskill, "Quantum hair," Phys. Scripta T36 (1991) 258-264. 


\section{Erratum}

The convention for the gauge coupling introduced in Sec. 2.4 was appropriate when all the charged particles known to the low energy observers have integer multiples of the smallest positive charge known. For more general cases, I use the convention for the gauge coupling described below.

I first define the unit charge as the largest positive charge with which all the charges of the particles known to the low energy observers become integers. Here, I assumed that the charges are quantized and this is always possible. I also assume that if a charged particle is known to the low energy observers, they take it for granted that there exists corresponding anti-particle with the opposite charge due to the CPT theorem. Then, since for every known charge $q$, there exists the opposite charge $-q$, whether a charge is positive or negative is a matter of convention. Then, as would be expected from the nomenclature, I use the normalization convention of the gauge coupling in which the unit charge is one.

The normalization convention of the gauge coupling I chose is convenient for describing the Dirac quantization condition. Indeed, with the above convention, it is an elementary exercise in number theory to show that there exists a combination of charged particles known to the low energy observers whose total charge is one. Then, the condition that the Dirac string should not be observable by the Aharonov-Bohm phase of such combination of charged particles going around the Dirac string gives the Dirac quantization condition:

$$
q_{m}=n,
$$

where $q_{m}$ is the magnetic charge of the monopole with the unit of the magnetic charge being $g_{m}=2 \pi / g$, and $n$ is some integer.

With my convention of the gauge coupling $g$ described above, the bound on the UV cut-off scale following from the magnetic WGC is given as

$$
\Lambda \lesssim g M_{P}
$$

It is straightforward to extend the proof given in Sec. 2.5 that the electric WGC is automatically satisfied when there exists a charged particle whose mass is below the UV cut-off scale (2.17) to more general cases discussed above. In more general cases, the UV cut-off scale following from the magnetic WGC is given by (A.8), with the convention of the gauge coupling $g$ explained above. The proof can be given following the similar line of reasoning given in (2.19), and noticing that all the absolute value of charges are greater or equal to one in the current convention of the gauge coupling. 